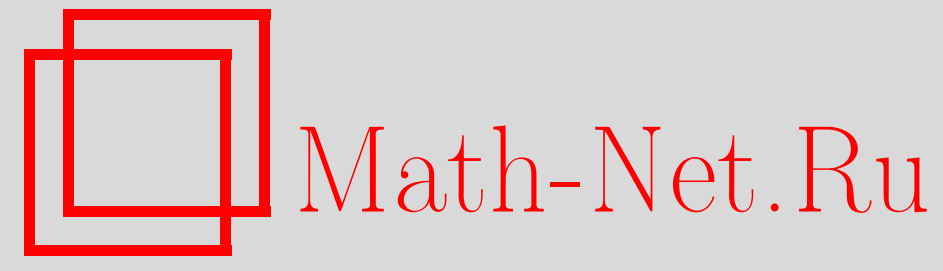

Е. Вл. Булинская, Полная классификация каталитических ветвящихся процессов, Теория вероятн. и ее примен., 2014, том 59, выпуск 4, 639-666

DOI: https://doi.org/10.4213/tvp4590

Использование Общероссийского математического портала Math-Net.Ru подразумевает, что вы прочитали и согласны с пользовательским соглашением http://www.mathnet.ru/rus/agreement

Параметры загрузки:

IP : 54.166 .219 .16

26 апреля 2023 г., 14:17:29

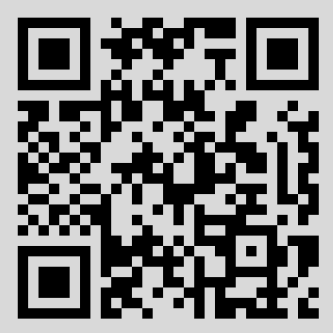




\title{
ПОЛНАЯ КЛАССИФИКАЦИЯ КАТАЛИТИЧЕСКИХ ВЕТВЯЩИХСЯ ПРОЦЕССОВ ${ }^{1)}$
}

\begin{abstract}
Изучается каталитический ветвящийся процесс (КВП) с произвольным конечным множеством катализаторов. Эта модель описывает систему частиц, в которой движение частиц задается марковской цепью с конечным или счетным пространством состояний, а ветвление может происходить только в точках нахождения катализаторов. Полученные результаты обобщают и усиливают известные ранее утверждения для КВП с одним катализатором и для ветвящегося случайного блуждания по $\mathbf{Z}^{d}, d \in \mathbf{N}$, с конечным числом источников размножения и гибели частиц. Предложена классификация КВП с $N$ катализаторами на надкритический, критический или докритический процессы в зависимости от значения перронова корня некоторой матрицы размера $N \times N$. Такая классификация согласуется с моментным анализом, проведенным для локальных и общих численностей частиц. Доказательства основных результатов базируются на построении вспомогательных многотипных процессов Беллмана-Харриса с привлечением времен достижения марковской цепью точки нахождения одного катализатора с запретом посещения остальных точек катализа, а также на применении многомерных теорем восстановления.
\end{abstract}

Ключевье слова и фразы: каталитический ветвящийся процесс, классификация, времена достижения с запретом, моментный анализ, многотипный процесс Беллмана-Харриса.

1. Введение. Рассматривается модель каталитического ветвящегося прочесса (КВП) с конечным множеством катализаторов. Она описывает систему частиц, перемещающихся в пространстве и производящих потомство только в присутствии катализаторов. Точнее говоря, пусть в момент $t=0$ имеется одна частица, движение которой по некоторому конечному или счетному множеству $S$ задается марковской цепью $\eta=\{\eta(t), t \geqslant 0\}$ с непрерывным временем и инфинитезимальной матрицей $Q=(q(x, y))_{x, y \in S}$. Когда частица достигает конечного множества катализаторов $W=\left\{w_{1}, \ldots, w_{N}\right\} \subset S$, скажем, в точке $w_{k}$, она проводит

* Московский государственный университет им. М. В. Ломоносова, Москва, Россия; bulinskaya@yandex.ru

1) Работа выполнена при поддержке фонда Дмитрия Зимина «Династия» и РФФИ (грант № 14-01-00318). 
там случайное время, имеющее экспоненциальное распределение с параметром $\beta_{k}>0$. Затем частица либо гибнет, либо покидает точку $w_{k}$ соответственно с вероятностями $\alpha_{k}$ и $1-\alpha_{k}\left(0 \leqslant \alpha_{k}<1\right)$. Если частица умирает (в точке $w_{k}$ ), то в момент гибели она замещается случайным числом $\xi_{k} \geqslant 0$ потомков, расположенных в той же точке $w_{k}$. Если частица покидает $w_{k}$, то она совершает скачок в точку $y \neq w_{k}$ с вероятностью $-q\left(w_{k}, y\right) q\left(w_{k}, w_{k}\right)^{-1}$ и продолжает движение согласно марковской цепи $\eta$. Предполагается, что все новорожденные частицы ведут себя как независимые копии родительской частицы.

Будем предполагать, что марковская цепь $\eta$ является неразложимой, а матрица $Q$ - консервативной (другими словами, $\sum_{y \in S} q(x, y)=0$, где $q(x, y) \geqslant 0$ при $x \neq y$ и $q(x, x) \in(-\infty, 0)$ для любого $x \in S)$. Обозначим $f_{k}(s):=\mathbf{E} s^{\xi_{k}}, s \in[0,1]$, вероятностную производящую функцию случайной величины $\xi_{k}, k=1, \ldots, N$. Будем придерживаться стандартного предположения существования конечной производной $f_{k}^{\prime}(1)$, т.е. конечности $\mathbf{E} \xi_{k}$ для любого $k=1, \ldots, N$.

Изучение КВП с одним катализатором было начато еще в XX веке (см., например, [12]). В этой связи отметим также недавнюю работу [21], где в качестве основных методов при выполнении моментного анализа процесса выступали спинальная техника, т.е. лемма «от многих к одному», и теория восстановления. Заметим, что обобщение упомянутых результатов на произвольное конечное множество катализаторов нетривиально, поскольку между катализаторами существует «конкуренция». Важный частный случай нескольких катализаторов (для которого $S=\mathbf{Z}^{d}, d \in \mathbf{N}$, и марковская цепь $\eta$ является симметричным пространственно-однородным случайным блужданием с конечной дисперсией скачков) был исследован в [34]. Там с помощью изучения спектральных свойств эволюционных операторов были получены достаточные условия экспоненциального роста численностей частиц. Мы следуем иному пути, позволяющему установить более точные результаты.

В рамках ветвящихся процессов естественная классическая задача заключается в анализе асимптотического поведения (при $t \rightarrow \infty)$ локальных и общих численностей частиц в момент $t$ (в отношении разнообразных ветвящихся процессов без катализаторов см., например, [8]). Пусть $\mu(t)$ обозначает общее число частиц, существующих в КВП в момент $t \geqslant 0$. Аналогично определим локальные численности $\mu(t ; y)$ как количества частиц, расположенных в различных точках $y \in S$ в момент $t$. В настоящей статье наша цель является троякой. Во-первых, мы предлагаем классификацию КВП (с $N$ катализаторами), рассматривая процесс как надкритический, критический или докритический, если перронов корень $\rho$ некоторой матрицы размера $N \times N$ с неотрицательными элементами больше, равен или меньше 1 соответственно. Во-вторых, мы проводим моментный анализ локальных и общих численностей ча- 
стиц, чтобы подтвердить естественность предложенной классификации (в самом деле, можно увидеть, что асимптотическое поведение моментов всех порядков существенным образом определяется введенным классом КВП). В-третьих, мы рассматриваем некоторые частные случаи КВП и тем самым показываем, что установленные результаты не только обобщают соответствующие результаты других работ, но даже уточняют их.

Наш подход состоит в использовании времен достижения марковской цепью точки нахождения одного катализатора с запретами посещения остальных точек катализа (см., например, [1], [9, гл. 2, § 11] и [17]) и построении вспомогательных ветвящихся процессов Беллмана-Харриса с не более чем $N(N+1)+1$ типами частиц. Такой метод инициирован в статье [32], где ветвящееся случайное блуждание по $\mathbf{Z}$ с одним катализатором было исследовано с помощью введения времен достижения (без запретов) точки нахождения катализатора и рассмотрения подходящего процесса Беллмана-Харриса с двумя типами частиц. Отметим также, что в [18] для изучения ветвящегося случайного блуждания по $\mathbf{Z}$ с дискретным временем и несколькими катализаторами авторы рассматривали вложенный многотипный ветвящийся процесс ГальтонаВатсона, получающийся в результате «забывания/выбрасывания времени, проведенного между посещениями мест расположения катализаторов». Последний подход является плодотворным при классификации ветвящегося случайного блуждания, поскольку многотипные процессы Гальтона-Ватсона и Беллмана-Харриса допускают одни и те же надкритический, критический или докритический режимы. Однако для дальнейшего изучения ветвящихся случайных блужданий (моментный анализ, предельные теоремы и т.п.) многотипного процесса ГальтонаВатсона не достаточно, и поэтому Кармона и Ху опирались в [18] на другую технику, такую как леммы «от многих к одному». Наш первый вспомогательный процесс строится таким образом, чтобы исследование КВП могло быть в основном сведено к анализу процесса БеллманаХарриса. Число частиц в этом процессе выбирается так, чтобы гарантировать неразложимость процесса. Оно не может быть меньше чем $N$ и не должно быть больше чем $N(N+1)$. Хорошо известно (см., например, [8, гл. $4, \S \S 5,6$ и 7]), что неразложимый многотипный процесс БеллманаХарриса классифицируется как надкритический, критический или докритический в зависимости от значения перронова корня матрицы средних числа непосредственных потомков частиц различных типов. Именно эта идея и лежит в основе классификации КВП. Кроме того, приводимая далее лемма 1 дает даже более удобную для проверки (поскольку размерность используемой матрицы понижается) форму классификации КВП с помощью перронова корня определенной неразложимой матрицы размера $N \times N$. Еще один вспомогательный процесс Беллмана-Харриса 
необходим при изучении общих численностей частиц в КВП в случае невозвратной марковской цепи $\eta$. Такой процесс выбирается разложимым с финальным типом частиц. Анализ вспомогательных ветвящихся процессов Беллмана-Харриса позволяет не только классифицировать КВП, но также вывести систему уравнений восстановления для средних локальных и общих численностей частиц в КВП. Чтобы осуществить моментный анализ локальных и общих численностей частиц, мы опираемся на многомерные теоремы восстановления, установленные в [19] и [28].

Используемый нами подход имеет преимущества, поскольку теория многотипных процессов Беллмана-Харриса хорошо развита и ее результаты могут быть применены к вспомогательным процессам БеллманаХарриса, что приводит к новым результатам для КВП. Укажем лишь несколько недавних работ, относящихся к многотипным процессам Беллмана-Харриса, например, [5], [25] и [33].

Заметим также, что КВП может трактоваться как марковский ветвящийся процесс с не более чем счетным числом типов частиц, поскольку положение частицы может быть ассоциировано с ее типом. Теория ветвящихся процессов со счетным числом типов частиц, несмотря на свою длительную историю (см., например, [29]), не была до сих пор систематизирована ввиду своей сложности. Некоторые новые работы, такие как [13], [14], [23] и [30], вносят важный вклад в это направление исследований. Однако результаты упомянутых работ не покрывают утверждения, установленные в данной статье. Несколько иные модели, описывающие перемещение и размножение частиц, исследовались в работах [7], [15] и [24]. Ветвящиеся случайные блуждания, изученные в этих статьях, пространственно-однородны, в то время как главной особенностью КВП является именно пространственная неоднородность. Некоторые различия в поведении однородного ветвящегося случайного блуждания по $\mathbf{Z}$ и его каталитического аналога обсуждаются в [18]. Завершая введение, отметим тесную связь каталитических ветвящихся случайных блужданий по $\mathbf{Z}$ и супер-броуновского движения с одним катализатором (см., например, [20] и [22]).

Опишем структуру статьи. В разделе 2 вводится первый вспомогательный процесс Беллмана-Харриса и предлагается классификация КВП. Раздел 3 включает теорему 1 с доказательством, представляющим моментный анализ локальных и общих численностей частиц в КВП. Там же мы строим второй вспомогательный процесс Беллмана-Харриса, необходимый при изучении общих численностей частиц в случае, когда марковская цепь $\eta$ невозвратна. Раздел 4 демонстрирует применения наших результатов к каталитическому ветвящемуся случайному блужданию по $\mathbf{Z}^{d}$ и к ветвящемуся процессу с одним катализатором. Там также приводится подробное сравнение наших результатов с известными ранее. 


\section{2. Вспомогательный процесс Беллмана-Харриса. Напо-} мним определение ветвящегося процесса Беллмана-Харриса с $n$ типами частиц. Процесс начинается с одной частицы типа $i \in\{1, \ldots, n\}$. Родительская частица имеет случайную продолжительность жизни с функцией распределения $G_{i}(t), t \geqslant 0$. Погибая, частица производит потомков в соответствии с вероятностной производящей функцией $g_{i}(\mathbf{s})$, $\mathbf{s}=\left(s_{1}, \ldots, s_{n}\right) \in[0,1]^{n}$. Новые частицы типа $j=1, \ldots, n$ эволюционируют независимо друг от друга и от предыстории процесса с распределением продолжительности жизни $G_{j}(\cdot)$ и производящей функцией числа потомков $g_{j}(\cdot)$. Обозначим

$$
M:=\left(m_{i, j}\right)_{i, j=1}^{n}, \quad \text { где } m_{i, j}=\left.\partial_{s_{j}} g_{i}\right|_{\mathbf{s}=(1, \ldots, 1)},
$$

матричу средних числа непосредственных потомков частии различных типов. Ветвящийся процесс Беллмана-Харриса называется неразложимылм, если матрица $M$ является неразложимой (необходимые сведения о неразложимых матрицах см., например, в [31, гл. $1, \S 3])$. Пусть $\rho$ - перронов корень матрицы $M$ (т.е. собственное значение с максимальным модулем). Процесс Беллмана-Харриса называется надкритическим, если $\rho>1$, критическим, если $\rho=1$, и докритическим, если $\rho<1$ (см., например, $[8$, гл. $4, \S \S 5,6$ и 7$])$. Обозначим $Z_{j}(t)$ число частиц типа $j$, существующих в момент $t$, где $t \geqslant 0, j=1, \ldots, n$.

Для построения вспомогательного процесса Беллмана-Харриса в рамках КВП нам требуется ввести некоторые обозначения. Рассмотрим частицу, перемешение которой по множеству $S$ определяется марковской цепью с генератором $Q$ и начальным состоянием $x$. Пусть ${ }_{H} \bar{\tau}_{x, y}$, где $x, y \in S$ и $H \subset S,-$ время, проведенное частицей после выхода из стартовой точки $x$ до первого попадания в $y$, если траектория частицы не проходит через множество $H$. В противном случае (если траектория частицы проходит через множество $H$ до первого попадания в $y$ ) полагаем ${ }_{H} \bar{\tau}_{x, y}=\infty$. Будем называть случайную величину ${ }_{H} \bar{\tau}_{x, y}$ временем достижения состояния $y$ с запретом в $H$ после первого выхода из стартового состояния $x$. Обозначим ${ }_{H} \bar{F}_{x, y}(t), t \geqslant 0$, несобственную функцию распределения ${ }_{H} \bar{\tau}_{x, y}$. Очевидно, что ${ }_{H} \bar{F}_{x, y}(0)=\left(\delta_{x, y}-1\right) q(x, y) q(x, x)^{-1}$, где $\delta_{x, y}$ есть символ Кронекера. Явные формулы для вероятности конечности величины ${ }_{H} \bar{\tau}_{x, y}$, т.е. для величины ${ }_{H} \bar{F}_{x, y}(\infty)=\lim _{t \rightarrow \infty} \bar{F}_{x, y}(t)$, вовлекающие вероятности с запретами и функцию Грина, выведены в [17]. Если множество запретов $H$ пусто, мы будем писать $\bar{\tau}_{x, y}$ и $\bar{F}_{x, y}(\cdot)$ вместо соответственно $\varnothing \bar{\tau}_{x, y}$ и $\varnothing \bar{F}_{x, y}(\cdot)$.

Вернемся к КВП. Временно предположим, что КВП стартует в $w_{p}$ для некоторого $p=1, \ldots, N$. Положим $W_{k}:=W \backslash\left\{w_{k}\right\}$ и для $j=1, \ldots, N$ обозначим $K_{j}:=\left\{k=1, \ldots, N:_{W_{k}} \bar{F}_{w_{j}, w_{k}}(\infty)-{ }_{W_{k}} \bar{F}_{w_{j}, w_{k}}(0)>0\right\}$. Пусть $K_{j}=\left\{k(j, 1), \ldots, k\left(j,\left|K_{j}\right|\right)\right\}$, где $1 \leqslant k(j, 1)<k(j, 2)<\cdots<k\left(j,\left|K_{j}\right|\right) \leqslant N$ 
и | · обозначает мощность конечного множества. Разделим популяцию частиц, существующих в момент $t \geqslant 0$, на $L+1$ групп при $L:=N+\sum_{j=1}^{N}\left|K_{j}\right|$. Частицы, находящиеся в момент $t$ в точке $w_{j}$, образуют $j$-ю группу, имеющую мощность $\mu\left(t ; w_{j}\right), j=1, \ldots, N$. Рассмотрим семейство, состоящее из частиц, которые покинули $w_{j}$ по крайней мере однажды за интервал $[0, t]$, а после последнего посещения $w_{j}$ еще не достигли $W$ к моменту $t$, но когда-нибудь попадут в $w_{k(j, i)}$ до возможного посещения множества $W_{k(j, i)}, i=1, \ldots,\left|K_{j}\right|, j=1, \ldots, N$. Это семейство имеет мощность, обозначаемую $\mu_{j, i}(t)$, и соответствует группе с номеpoм $L(j)+i$, где $L(j):=N+\sum_{l=1}^{j-1}\left|K_{l}\right|$. Группа номер $L+1$ содержит оставшиеся частицы, не включенные в первые $L$ групп. Заметим, что последняя группа состоит из частиц, имеющих бесконечную продолжительность жизни, поскольку после момента $t$ они больше никогда не попадут в множество катализаторов. Таким образом, после момента $t$ эти частицы не произведут потомков и не окажут влияния на численности частиц в других $L$ группах.

Теперь мы можем ввести вспомогательный процесс БеллманаХарриса, чтобы использовать его для изучения КВП. Рассмотрим $L$ мерный процесс Беллмана-Харриса, стартующий с одной частицы типа $p$ и имеющий следующие функции распределения и производящие функции:

$$
\begin{gathered}
G_{j}(t)=1-e^{-\beta_{j} t} \\
g_{j}(\mathbf{s})=\alpha_{j} f_{j}\left(s_{j}\right)+\left(1-\alpha_{j}\right) \sum_{k=1}^{N} W_{k} \bar{F}_{w_{j}, w_{k}}(0) s_{k} \\
+\left(1-\alpha_{j}\right) \sum_{i=1}^{\left|K_{j}\right|} T_{i, j}(\infty) s_{L(j)+i}+\left(1-\alpha_{j}\right)\left(1-\sum_{k=1}^{N} W_{k} \bar{F}_{w_{j}, w_{k}}(\infty)\right), \\
G_{L(j)+i}(t)=\frac{T_{i, j}(t)}{T_{i, j}(\infty)}, \quad g_{L(j)+i}(\mathbf{s})=s_{k(j, i)},
\end{gathered}
$$

где $T_{i, j}(t):={ }_{W_{k(j, i)}} \bar{F}_{w_{j}, w_{k(j, i)}}(t)-W_{k(j, i)} \bar{F}_{w_{j}, w_{k(j, i)}}(0), 0 \leqslant t \leqslant \infty$ и $i=$ $1, \ldots,\left|K_{j}\right|, j=1, \ldots, N$. Легко видеть, что для процесса, построенного таким образом, вектор $\left(Z_{1}(t), \ldots, Z_{L}(t)\right)$ для каждого $t \geqslant 0$ имеет такое же распределение, как и вектор размерности $L, j$-я координата которого есть $\mu\left(t ; w_{j}\right)$, а $(L(j)+i)$-я координата представляет собой $\mu_{j, i}(t)$, $i=1, \ldots,\left|K_{j}\right|, j=1, \ldots, N$.

Матрица средних $M=\left(m_{k, l}\right)_{k, l=1}^{L}$ введенного процесса БеллманаХарриса имеет блочный вид, т.е.

$$
M=\left(\begin{array}{cccc}
M_{1,1} & M_{1,2} & \ldots & M_{1, N+1} \\
M_{2,1} & M_{2,2} & \ldots & M_{2, N+1} \\
\ldots & \ldots & \ldots & \ldots \\
M_{N+1,1} & M_{N+1,2} & \ldots & M_{N+1, N+1}
\end{array}\right) .
$$


Здесь матрица $M_{1,1}=\left(m_{k, l}\right)_{k, l=1}^{N}$ обладает элементами $m_{k, l}=$ $\delta_{k, l} \alpha_{k} f_{k}^{\prime}(1)+\left(1-\alpha_{k}\right)_{W_{l}} \bar{F}_{w_{k}, w_{l}}(0)$. Для $j=1, \ldots, N$ все элементы $N \times\left|K_{j}\right|$ матрицы $M_{1, j+1}$ равны нулю, кроме $j$-й строки, а при $i=1, \ldots,\left|K_{j}\right|$ имеем $m_{j, L(j)+i}=\left(1-\alpha_{j}\right) T_{i, j}(\infty)$. Если $i=1, \ldots, N$, то $\left|K_{i}\right| \times N$-матрица $M_{i+1,1}$ такова, что, опуская ее $j$-е нулевые столбцы для всех $j \notin K_{i}$, получаем единичную матрицу размера $\left|K_{i}\right| \times\left|K_{i}\right|$. Если $i, j \in\{1, \ldots, N\}$, то $\left|K_{i}\right| \times\left|K_{j}\right|$-матрица $M_{i+1, j+1}$ содержит лишь нулевые элементы.

Проверим, что заданный процесс Беллмана-Харриса является неразложимым. В силу неразложимости марковской цепи $\eta$ существует конечный путь из $w_{k}$ в $w_{l}$ с положительной вероятностью для каждых $k, l=1, \ldots, N$. В рамках КВП такой путь имеет также положительную вероятность, поскольку, находясь в точке расположения катализатора $w_{i}$, частица может покинуть его без ветвления с положительной вероятностью $1-\alpha_{i}$ для каждого $i=1, \ldots, N$. Среди точек, посещенных последовательно этой траекторией марковской цепи $\eta$, выберем те, которые лежат в множестве $W$, скажем, $w_{p(0)}, w_{p(1)}, \ldots, w_{p(J)}, w_{p(J+1)}$ при $p(0):=k$ и $p(J+1):=l$. Согласно построению вспомогательного процесса Беллмана-Харриса такой путь соответствует изменениям типа частицы с $k$ на $l$. А именно, если путь цепи $\eta$ достигает $w_{p(j+1)}$ сразу после выхода из $w_{p(j)}$, то тип частицы в процессе Беллмана-Харриса меняется с $p(j)$ на $p(j+1)$. В противном случае, $p(j+1) \in K_{p(j)}$, т.е. $p(j+1)=k(p(j), i)$ для некоторого $i=1, \ldots,\left|K_{p(j)}\right|$, и изменение типа частицы с $p(j)$ на $p(j+1)$ содержит промежуточный тип $L(p(j))+i$. Следовательно, мы заключаем, что для каждых $k$ и $l$ из $\{1, \ldots, N\}$ существуют $n=n(k, l) \in \mathbf{N}$ и набор $\{r(1), \ldots, r(n)\} \subset\{1, \ldots, L\}$ такие, что $m_{k, r(1)} m_{r(1), r(2)} \cdots m_{r(n), l}>0$. Поскольку $m_{L(j)+i, k(j, i)}=1>0$ и $m_{j, L(j)+i}>0$ для каждых $i=1, \ldots,\left|K_{j}\right|$ и $j=1, \ldots, N$ согласно определению (3) матрицы $M$, то предыдущее утверждение остается также справедливым при $k, l=1, \ldots, L$. Тем самым, мы проверили, что для каждых $k$ и $l$ из $\{1, \ldots, L\}$ существует $n=n(k, l) \in \mathbf{N}$ такое, что $m_{k, l}^{(n)}>0$, где $m_{k, l}^{(n)}$ есть $(k, l)$-й элемент матрицы $M^{n}$. Таким образом, матрица $M$ неразложима. Более того, если $f_{i}^{\prime}(1)>0$ для некоторого $i=1, \ldots, N$, то $m_{i, i}>0$ и матрица $M$ непериодическая (см., например, [31, гл. $\left.\left.1, \S 2\right]\right)$. В этом случае $M$ является примитивной матрицей (см., например, [31, гл. $1, \S 3])$.

В силу теоремы Перрона-Фробениуса для неразложимых матриц (см., например, $[31$, гл. $1, \S 4]$ ) матрица $M$ обладает положительным собственным значением $\rho(M)$, которое имеет максимальный модуль среди всех собственных значений этой матрицы. Величина $\rho(M)$ называется перроновым корнем матрицы $M$. Используя классификацию построенного процесса Беллмана-Харриса, назовем КВП надкритическим, критическим или докритическим, если соответственно $\rho(M)>1, \rho(M)=1$ или $\rho(M)<1$, где матрица $M$ задается формулой $(3)$. 
Пусть $G_{i}^{*}(\lambda):=\int_{0-}^{\infty} e^{-\lambda t} d G_{i}(t), \lambda \geqslant 0$, - преобразование ЛапласаСтилтьеса функции распределения $G_{i}$, а $H(\lambda):=\left(G_{i}^{*}(\lambda) m_{i, j}\right)_{i, j=1}^{L}$. Отметим, что $H(0)=M$. Матрица $H(\lambda)$ является неразложимой ввиду неразложимости матрицы $M$. Положим также $D(\lambda)=\left(d_{i, j}(\lambda)\right)_{i, j=1}^{N}, \lambda \geqslant 0$, с $d_{i, j}(\lambda):=\delta_{i, j} \alpha_{i} f_{i}^{\prime}(1) G_{i}^{*}(\lambda)+\left(1-\alpha_{i}\right) G_{i}^{*}(\lambda)_{W_{j}} \bar{F}_{w_{i}, w_{j}}^{*}(\lambda)$. Легко видеть, что матрица $D=\left(d_{i, j}\right)_{i, j=1}^{N}$, определенная как $D:=D(0)$, имеет элементы

$$
d_{i, j}=\delta_{i, j} \alpha_{i} f_{i}^{\prime}(1)+\left(1-\alpha_{i}\right)_{W_{j}} \bar{F}_{w_{i}, w_{j}}(\infty) .
$$

Для неразложимой матрицы $A$ обозначим $\rho(A)$ ее перронов корень. Следующее утверждение дает удобное условие критичности для КВП.

Лемма 1. Для каждого $\lambda \geqslant 0$ матрица $D(\lambda)$ неразложима, $a$ значения $\rho(D(\lambda))$ и $\rho(H(\lambda))$ одновременно больше 1 , равнь 1 или меньше 1. В частности, перроновы корни $\rho(D)$ и $\rho(M)$ одновременно либо больше 1, либо равнь 1, либо меньше 1.

Д о к а з а т е л ь с т в о. Неразложимость матрицы $D$ (а следовательно, и матрицы $D(\lambda))$ устанавливается так же, как неразложимость матрицы $M$. Согласно теореме Перрона-Фробениуса матрица $H(\lambda)$ имеет строго положительный левый собственный вектор $\mathbf{v}$, соответствующий собственному значению $\rho(H(\lambda))$. Тем самым, $H(\lambda)^{\top} \mathbf{v}=\rho(H(\lambda)) \mathbf{v}$, где символ ${ }^{\top}$ обозначает транспонирование, а все векторы рассматриваются как векторы-столбцы. Другими словами, $\left(H(\lambda)^{\top}-\rho(H(\lambda)) I\right) \mathbf{v}=\mathbf{0}$, где $I$ - единичная матрица, а $\mathbf{0}$ - вектор размерности $L$ с нулевыми координатами. Применим некоторые равносильные преобразования к полученной системе уравнений, а именно, для каждых $j, k=1, \ldots, N$ прибавим $(L(j)+i)$-ю строку, умноженную на $G_{L(j)+i}^{*}(\lambda) \rho(H(\lambda))^{-1}$, к $k$-й строке, если $k \in K_{j}$, т.е. $k=k(j, i)$ для некоторого $i=1, \ldots,\left|K_{j}\right|$. Учитывая только первые $N$ уравнений, мы заключаем, что

$$
\mathbf{v}_{0}^{\top}\left(H_{1,1}(\lambda)+\rho(H(\lambda))^{-1} R(\lambda)-\rho(H(\lambda)) I\right)=\mathbf{0}^{\top} .
$$

Здесь $H_{1,1}(\lambda):=\left(G_{i}^{*}(\lambda) m_{i, j}\right)_{i, j=1}^{N}$, матрица $R(\lambda):=\left(r_{i, j}(\lambda)\right)_{i, j=1}^{N}$ имеет эле-

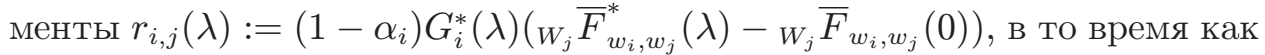
$\mathbf{v}_{0}$ есть строго положительный вектор, координаты которого совпадают с первыми $N$ координатами вектора $\mathbf{v}$, а $\mathbf{0}$ - вектор размерности $N$ с нулевыми координатами. Заметим, что $D(\lambda)=H_{1,1}(\lambda)+R(\lambda)$. Очевидно, что если $\rho=\rho(H(\lambda)) \geqslant 1$, то

$$
\begin{aligned}
\rho^{-1} \mathbf{v}_{0}^{\top}\left(D(\lambda)-\rho^{2} I\right) & =\mathbf{v}_{0}^{\top}\left(\rho^{-1} H_{1,1}(\lambda)+\rho^{-1} R(\lambda)-\rho I\right) \\
& \leqslant \mathbf{v}_{0}^{\top}\left(H_{1,1}(\lambda)+\rho^{-1} R(\lambda)-\rho I\right) \\
& \leqslant \mathbf{v}_{0}^{\top}\left(H_{1,1}(\lambda)+R(\lambda)-\rho I\right)=\mathbf{v}_{0}^{\top}(D(\lambda)-\rho I) .
\end{aligned}
$$

Аналогично, если $\rho(H(\lambda)) \leqslant 1$, то предыдущая цепочка неравенств остается справедливой при замене каждого символа $\leqslant$ на $\geqslant$. Окончательно 
получаем

$$
\begin{aligned}
& D(\lambda)^{\top} \mathbf{v}_{0} \geqslant \rho(H(\lambda)) \mathbf{v}_{0} \quad \text { и } \quad D(\lambda)^{\top} \mathbf{v}_{0} \leqslant \rho(H(\lambda))^{2} \mathbf{v}_{0} \quad \text { при } \rho(H(\lambda)) \geqslant 1, \\
& D(\lambda)^{\top} \mathbf{v}_{0} \leqslant \rho(H(\lambda)) \mathbf{v}_{0} \quad \text { и } \quad D(\lambda)^{\top} \mathbf{v}_{0} \geqslant \rho(H(\lambda))^{2} \mathbf{v}_{0} \quad \text { при } \rho(H(\lambda)) \leqslant 1 .
\end{aligned}
$$

В силу теоремы 1.6 из [31, гл. $1, \S 4]$ второе соотношение в (5) и первое в (6) влекут за собой, что $\rho(D(\lambda)) \leqslant \rho(H(\lambda))^{2}$ при $\rho(H(\lambda)) \geqslant 1$ и $\rho(D(\lambda)) \leqslant \rho(H(\lambda))$ при $\rho(H(\lambda)) \leqslant 1$. Учитывая доказательство теоремы Перрона-Фробениуса, благодаря первому неравенству в (5) и второму в (6) выводим, что $\rho(D(\lambda)) \geqslant \rho(H(\lambda))$ при $\rho(H(\lambda)) \geqslant 1$ и $\rho(D(\lambda)) \geqslant \rho(H(\lambda))^{2}$ при $\rho(H(\lambda)) \leqslant 1$. Следовательно, если $\rho(H(\lambda)) \geqslant 1$, то $\rho(H(\lambda)) \leqslant \rho(D(\lambda)) \leqslant \rho(H(\lambda))^{2}$, в то время как в случае $\rho(H(\lambda)) \leqslant 1$ мы имеем $\rho(H(\lambda))^{2} \leqslant \rho(D(\lambda)) \leqslant \rho(H(\lambda))$. Из этих оценок вытекает утверждение леммы 1.

Установленная лемма позволяет следующим образом переформулировать определение надкритического, критического и докритического КВП с помощью матрицы $D$.

О п р е д е л е н и е 1 . КВП называется надкритическим, критическим или докритическим, если соответственно $\rho(D)>1, \rho(D)=1$ или $\rho(D)<1$, где матрица $D$ задается формулой $(4)$.

3. Моментный анализ КВП. Мод показал (см. [27]), что математические ожидания численностей частиц различных типов в надкритическом неразложимом многотипном процессе Беллмана-Харриса растут экспоненциально (когда время стремится к бесконечности) с некоторой скоростью $\nu$, называемой мальтусовским параметром. Параметр $\nu$ положителен и определяется как единственное решение уравнения $\rho(H(\nu))=1$. Лемма 1 показывает, что для нашего вспомогательного $L$-мерного надкритического процесса Беллмана-Харриса эта процедура упрощается и мальтусовский параметр может быть найден как единственный корень уравнения $\rho(D(\nu))=1$ (заметим, что квадратная матрица $H(\lambda)$ имеет порядок $L \geqslant N$, в то время как квадратная матрица $D(\lambda)$ имеет порядок $N)$. Таким образом, если $\rho(D)>1$, то в рамках КВП мальтусовский параметр $\nu$ задается как единственный (положительный) корень уравнения $\rho(D(\nu))=1$. Если $\rho(D)=\rho(D(0))>1$, то такое решение всегда существует. Действительно, согласно теореме Перрона-Фробениуса функция $\rho(D(\lambda))$ по отношению к переменной $\lambda$ является строго убывающей и стремится к 0 при $\lambda \rightarrow \infty$.

Чтобы сформулировать основной результат статьи, нам потребуются некоторые новые обозначения. Если $R$ - матрица размера $n \times n$, то $R_{i, j}$ обозначает матрицу размера $(n-1) \times(n-1)$, получающуюся в результате удаления $i$-й строки и $j$-го столбца матрицы $R, i, j=1, \ldots, n$. Для надкритического КВП рассмотрим функции 
$a_{n}(x, y), x, y \in S, n \in \mathbf{N}$, которые возникнут в описании асимптотического поведения (при $t \rightarrow \infty) n$-х факториальных моментов $m_{n}(t ; x, y):=\mathbf{E}_{x} \mu(t ; y)(\mu(t ; y)-1) \cdots(\mu(t ; y)-n+1)$ локальных численностей частиц в КВП (индекс $x$ обозначает стартовую точку КВП). Функция $a_{1}(x, y)$ имеет наиболее простой вид, когда $x, y \in W$, а именно,

$$
a_{1}\left(w_{i}, w_{j}\right)=\frac{\Delta_{j, i}(\nu)}{\left(\nu+\beta_{j}\right) \Delta^{\prime}(\nu)}, \quad i, j=1, \ldots, N,
$$

где $\Delta_{i, j}(\lambda):=(-1)^{i+j} \operatorname{det}(I-D(\lambda))_{i, j}$ и $\Delta^{\prime}(\nu):=\left.\frac{d}{d \lambda} \operatorname{det}(I-D(\lambda))\right|_{\lambda=\nu}$. Если $N=1$, то функция $\Delta_{1,1}(\lambda), \lambda \geqslant 0$, полагается тождественно равной 1. Для $y \in S \backslash W$ пусть

$$
a_{1}\left(w_{i}, y\right)=\frac{\sum_{j=1}^{N} \Delta_{j, i}(\nu)\left(1-\alpha_{j}\right) G_{j}^{*}(\nu)_{W} \bar{F}_{w_{j}, y}^{*}(\nu)}{(\nu-q(y, y)) \Delta^{\prime}(\nu)\left(1-{ }_{W} F_{y, y}^{*}(\nu)\right)}, \quad i=1, \ldots, N
$$

Здесь ${ }_{H} F_{x^{\prime}, y^{\prime}}^{*}(\lambda):=\left(-q\left(x^{\prime}, x^{\prime}\right)\right)\left(\lambda-q\left(x^{\prime}, x^{\prime}\right)\right)^{-1}{ }_{H} \bar{F}_{x^{\prime}, y^{\prime}}^{*}(\lambda), \lambda \geqslant 0, x^{\prime}, y^{\prime} \in S$, $H \subset S$, т.е. ${ }_{H} F_{x^{\prime}, y^{\prime}}(t), t \geqslant 0$, - это функция распределения времени достижения состояния $y^{\prime}$ с запретом в $H$, если марковская цепь $\eta$ стартовала из состояния $x^{\prime}$ (см., например, [17]). Отметим, что $G_{j}^{*}(\lambda)=$ $\beta_{j}\left(\lambda+\beta_{j}\right)^{-1}$ для каждого $\lambda \geqslant 0$ и $j=1, \ldots, N$. Значения $a_{n}(x, y)$ при $n>1, x \in W$ и $y \in S$ вычисляются с помощью следующей рекуррентной схемы:

$$
a_{n}\left(w_{i}, y\right)=\sum_{k=1}^{N} \frac{\beta_{k} \Delta_{k, i}(n \nu)}{\left(n \nu+\beta_{k}\right) \Delta(n \nu)} h_{n, k}\left(a_{1}\left(w_{k}, y\right), \ldots, a_{n-1}\left(w_{k}, y\right)\right),
$$

где $i=1, \ldots, N, \Delta(\lambda):=\operatorname{det}(I-D(\lambda)), \lambda \geqslant 0$,

$$
h_{n, k}\left(z_{1}, \ldots, z_{n-1}\right):=\alpha_{k} \sum_{r=2}^{n} \frac{f_{k}^{(r)}(1)}{r !} \sum_{\substack{i_{1}, \ldots, i_{r}>0 \\ i_{1}+\cdots+i_{r}=n}} \frac{n !}{i_{1} ! \cdots i_{r} !} z_{i_{1}} \cdots z_{i_{r}}
$$

и $k=1, \ldots, N$. На этом пути значения $a_{n}(x, y)$ при $n \geqslant 1, x \in S \backslash W$ и $y \in S$ определяются согласно формуле

$$
a_{n}(x, y)=\sum_{i=1}^{N} W_{i} F_{x, w_{i}}^{*}(n \nu) a_{n}\left(w_{i}, y\right) .
$$

Определим функции $A_{n}(x), x \in S, n \in \mathbf{N}$, появляющиеся в асимптотических формулах для $n$-х факториальных моментов

$$
M_{n}(t ; x):=\mathbf{E}_{x} \mu(t)(\mu(t)-1) \cdots(\mu(t)-n+1)
$$

общих численностей частиц в надкритическом КВП. А именно, для $n=1$ и $x \in W$ положим

$$
A_{1}\left(w_{i}\right)=\sum_{j=1}^{N} \frac{\alpha_{j} \beta_{j}\left(f_{j}^{\prime}(1)-1\right) \Delta_{j, i}(\nu)}{\nu\left(\nu+\beta_{j}\right) \Delta^{\prime}(\nu)}, \quad i=1, \ldots, N .
$$


Если $n>1$ и $x \in W$, то значения $A_{n}(x)$ находятся в соответствии со следующей рекуррентной схемой:

$$
A_{n}\left(w_{i}\right)=\sum_{j=1}^{N} \frac{\beta_{j} \Delta_{j, i}(n \nu)}{\left(n \nu+\beta_{j}\right) \Delta(n \nu)} h_{n, j}\left(A_{1}\left(w_{j}\right), \ldots, A_{n-1}\left(w_{j}\right)\right) .
$$

Опираясь на последние равенства, можно получить значения $A_{n}(x)$ при $n \geqslant 1$ и $x \in S \backslash W$ с помощью соотношения

$$
A_{n}(x)=\sum_{i=1}^{N} W_{i} F_{x, w_{i}}^{*}(n \nu) A_{n}\left(w_{i}\right)
$$

Для критического КВП введем функции $b_{n}(x, y)$ и $B_{n}(x), x, y \in S$, $n \in \mathbf{N}$, возникающие в асимптотических соотношениях соответственно для функций $m_{n}(t ; x, y)$ и $M_{n}(t ; x)$. А именно, для $i, j=1, \ldots, N$ и $y \in$ $S \backslash W$ пусть

$$
b_{1}\left(w_{i}, w_{j}\right)=\frac{\Delta_{j, i}(0)}{\beta_{j} \Delta^{\prime}(0)}, \quad b_{1}\left(w_{i}, y\right)=\frac{\sum_{j=1}^{N} \Delta_{j, i}(0)\left(1-\alpha_{j}\right)_{W} F_{w_{j}, y}(\infty)}{-q(y, y) \Delta^{\prime}(0)\left(1-{ }_{W} F_{y, y}(\infty)\right)},
$$

где $\Delta^{\prime}(0):=\left.\frac{d}{d \lambda} \operatorname{det}(I-D(\lambda))\right|_{\lambda=0+}$. Если $n>1, i=1, \ldots, N$ и $y \in S$, то значения $b_{n}\left(w_{i}, y\right)$ вычисляются согласно рекуррентной схеме

$$
b_{n}\left(w_{i}, y\right)=\sum_{k=1}^{N} \frac{\alpha_{k} f_{k}^{\prime \prime}(1) \Delta_{k, i}(0)}{2(n-1) \Delta^{\prime}(0)} \sum_{r=1}^{n-1}\left(\begin{array}{l}
n \\
r
\end{array}\right) b_{r}\left(w_{k}, y\right) b_{n-r}\left(w_{k}, y\right) .
$$

Наконец, значения $b_{n}(x, y)$ при $n \geqslant 1, x \in S \backslash W$ и $y \in S$ определяются формулой

$$
b_{n}(x, y)=\sum_{i=1}^{N} W_{i} F_{x, w_{i}}(\infty) b_{n}\left(w_{i}, y\right)
$$

Для $i=1, \ldots, N$ положим

$$
B_{1}\left(w_{i}\right)=\sum_{j=1}^{N} \frac{\alpha_{j}\left(f_{j}^{\prime}(1)-1\right) \Delta_{j, i}(0)}{\Delta^{\prime}(0)} .
$$

Если $n>1$, то значения $B_{n}\left(w_{i}\right)$ подсчитываются рекуррентно, а именно,

$$
B_{n}\left(w_{i}\right)=\sum_{j=1}^{N} \frac{\alpha_{j} f_{j}^{\prime \prime}(1) \Delta_{j, i}(0)}{2(2 n-1) \Delta^{\prime}(0)} \sum_{r=1}^{n-1}\left(\begin{array}{l}
n \\
r
\end{array}\right) B_{r}\left(w_{j}\right) B_{n-r}\left(w_{j}\right), \quad i=1, \ldots, N .
$$

На основе последних равенств значения $B_{n}(x)$ при $n \geqslant 1$ и $x \in S \backslash W$ находятся следующим образом:

$$
B_{n}(x)=\sum_{i=1}^{N} W_{i} F_{x, w_{i}}(\infty) B_{n}\left(w_{i}\right)
$$


Наконец, для докритического КВП определим функции $C_{n}(x), x \in S$, $n \in \mathbf{N}$, появляющиеся в описании асимптотического поведения $M_{n}(t ; x)$. Пусть

$$
\begin{gathered}
C_{1}\left(w_{i}\right)=1+\sum_{j=1}^{N} \frac{\alpha_{j}\left(f_{j}^{\prime}(1)-1\right) \Delta_{j, i}(0)}{\Delta(0)}, \quad i=1, \ldots, N, \\
C_{1}(x)=1-\sum_{i=1}^{N} W_{i} F_{x, w_{i}}(\infty)+\sum_{i=1}^{N} W_{i} F_{x, w_{i}}(\infty) C_{1}\left(w_{i}\right), \quad x \in S \backslash W,
\end{gathered}
$$

а для $n>1$ положим

$$
\begin{gathered}
C_{n}\left(w_{i}\right)=\sum_{j=1}^{N} \frac{\Delta_{j, i}(0)}{\Delta(0)} h_{n, j}\left(C_{1}\left(w_{j}\right), \ldots, C_{n-1}\left(w_{j}\right)\right), \quad i=1, \ldots, N, \\
C_{n}(x)=\sum_{i=1}^{N} W_{i} F_{x, w_{i}}(\infty) C_{n}\left(w_{i}\right), \quad x \in S \backslash W .
\end{gathered}
$$

Следующая теорема является основным результатом настоящего раздела, в котором проводится моментный анализ локальных и общих численностей частиц в КВП. Эта теорема обобщает теоремы 4.1, 4.2 из [10] и теорему 1 из [21], а также некоторые утверждения теоремы 2 из [12].

Теорема 1. Для каждого $n \in \mathbf{N}$ при условии $\mathbf{E} \xi_{i}^{n}<\infty$ для любого $i=1, \ldots, N$ функиии $m_{n}(t ; x, y)$ и $M_{n}(t ; x)$ ограничень на произвольном конечном интервале из $[0, \infty)$ при фиксированных $x, y \in S$. Более того, асимптотическое поведение $m_{n}(t ; x, y)$ и $M_{n}(t ; x)$ при $t \rightarrow \infty$ зависит существенно от класса КВП и описььвается следуюшим образом.

1. $\operatorname{Ecли~} \rho(D)>1$, mо $\nu>0$ и при $t \rightarrow \infty$

$$
\begin{aligned}
m_{n}(t ; x, y) & =a_{n}(x, y) e^{n \nu t}+o\left(e^{n \nu t}\right), \\
M_{n}(t ; x) & =A_{n}(x) e^{n \nu t}+o\left(e^{n \nu t}\right),
\end{aligned}
$$

где функиии $a_{n}(x, y)$ и $A_{n}(x), x, y \in S$, строго положительнь.

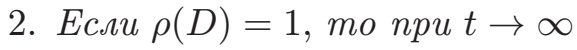

$$
\begin{aligned}
m_{n}(t ; x, y) & =b_{n}(x, y) t^{n-1}+o\left(t^{n-1}\right), \\
M_{n}(t ; x) & =B_{n}(x) t^{2 n-1}+o\left(t^{2 n-1}\right),
\end{aligned}
$$

где функиия $b_{n}(x, y)$ строго положительна при всех значениях $x, y \in S$ тогда и только тогда, когда $\int_{0}^{\infty} u d_{W_{j}} \bar{F}_{w_{i}, w_{j}}(u)<\infty$ при любьх $i, j=$ $1, \ldots, N$ и, дополнительно, в случае возвратной чепи $\eta$ и $n \geqslant 2$ верно неравенство $\sum_{i=1}^{N} \alpha_{i}\left|f_{i}(s)-s\right|>0$ для некоторого $s \in[0,1)$. Далее, функиия $B_{n}(x)$ строго положительна для любого $x \in S$ тогда и только тогда, когда марковская иепь $\eta$ невозвратна $u \int_{0}^{\infty} u d_{W_{j}} \bar{F}_{w_{i}, w_{j}}(u)<\infty$ для 
всех $i, j=1, \ldots, N$. B противном случае соответственно $b_{n}(\cdot, \cdot) \equiv 0$ и $B_{n}(\cdot) \equiv 0$.

3. Если $\rho(D)<1$, то при $t \rightarrow \infty$

$$
\begin{aligned}
m_{n}(t ; x, y) & =o(1), \\
M_{n}(t ; x) & =C_{n}(x)+o(1),
\end{aligned}
$$

где функиия $C_{n}(x)$ строго положительна для каждого $x \in S$ тогда $и$ только тогда, когда марковская иепь $\eta$ невозвратна. $B$ противном cлучае $C_{n}(\cdot) \equiv 0$.

Подчеркнем, что мы не налагаем на характер марковской цепи $\eta$ таких ограничений, как симметрия и однородность переходных вероятностей или конечная дисперсия скачков и т.п. Поэтому в теореме 1 мы устанавливаем точные асимптотические соотношения в пункте 1 и только частично в пунктах 2 и 3 . Невозможно предоставить какуюлибо полезную и общую информацию по пунктам 2 и 3 без дальнейших предположений о лежащем в основе КВП движении частиц. Например, даже для критического симметричного ветвящегося случайного блуждания по $\mathbf{Z}^{d}$ с одним катализатором (см. [12]) имеется четыре различных асимптотических формулы для $m_{n}(t ; x, y)$ и $M_{n}(t ; x)$ в зависимости от размерности $d=1,2,3$ или 4 и, тем самым, в зависимости от скорости убывания переходных вероятностей (заметим, что для $d \geqslant 5$ теорема 1 обобщает соответствующие результаты из [12]). Более того, для докритического ветвящегося случайного блуждания по $\mathbf{Z}^{d}$ с одним катализатором (см. [2]) порядки убывания функций $m_{n}(t ; x, y)$ отличаются для Bcex $d \in \mathbf{N}$.

Перейдем к проверке справедливости утверждений, сформулированных в теореме 1 .

Д о к а з а т е л с т в о. Главным образом, мы будем использовать вспомогательный процесс Беллмана-Харриса, построенный в разделе 2. Вначале установим теорему 1 для $x, y \in W$ (случай 1 ) и далее распространим эти результаты на общий случай $x, y \in S$ (случай 2 ).

Случай 1. Итак, предположим, что $x, y \in W$. Дальнейшее доказательство теоремы 1 для случая 1 разбивается на 9 шагов. На шаге 1 мы выводим систему уравнений восстановления относительно факториальных моментов локальных численностей частиц в КВП. Затем на шагах 2, 3 и 4 мы опираемся на эту систему, чтобы доказать соответствующие утверждения $(25),(27)$ и $(29)$ теоремы 1 , относящиеся к моментному анализу локальных численностей частиц. Далее, на шагах 5 и 6 выводим систему уравнений восстановления относительно факториальных моментов общих численностей частиц в КВП в зависимости от того, является марковская цепь $\eta$ возвратной или невозвратной. Наконец, на шагах 7, 8 и 9 мы пользуемся этими системами, чтобы установить соответственно 
утверждения (26), (28) и (30) теоремы 1, касающиеся моментного анализа общего числа частиц. Заметим, что на шаге 6 мы вводим вспомогательный разложимый $(L+1)$-мерный процесс Беллмана-Харриса с финальным типом частиц.

Ш а г 1. На данной стадии выведем систему уравнений восстановления относительно факториальных моментов локальных численностей частиц в КВП. При $t \geqslant 0, n \in \mathbf{N}$ и $k, l=1, \ldots, L$ обозначим $m_{n}^{\mathrm{BH}}(t ; k, l):=$ $\mathbf{E}_{k} Z_{l}(t)\left(Z_{l}(t)-1\right) \cdots\left(Z_{l}(t)-n+1\right)$ факториальный момент $n$-го порядка числа частиц $Z_{l}(t)$ типа $l$ в момент $t$ в процессе Беллмана-Харриса (индекс $k$ означает тип родительской частицы). В соответствии с построением вспомогательного процесса имеем $m_{n}\left(t ; w_{i}, w_{j}\right)=m_{n}^{\mathrm{BH}}(t ; i, j)$ для всех $n \in \mathbf{N}, t \geqslant 0, i, j=1, \ldots, N$. Таким образом, если выполнено условие $\mathbf{E} \xi_{k}^{n}<\infty$ для некоторого $n \in \mathbf{N}$ и всех $k=1, \ldots, N$, то конечность функций $m_{n}\left(t ; w_{i}, w_{j}\right)$ для каждого $t \geqslant 0$ и любых $i, j=1, \ldots, N$ вытекает из теоремы 1 в $[8$, гл. 8, $\S 6]$. Более того, в силу этой теоремы функции $m_{1}^{\mathrm{BH}}(t ; k, l), t \geqslant 0, k, l=1, \ldots, L$, удовлетворяют следующей системе уравнений восстановления:

$$
m_{1}^{\mathrm{BH}}(t ; k, l)=\delta_{k, l}\left(1-G_{k}(t)\right)+\sum_{r=1}^{L} m_{k, r} \int_{0}^{t} m_{1}^{\mathrm{BH}}(t-u ; r, l) d G_{k}(u)
$$

в то время как при $n>1$ эта теорема и обобщенная формула Фаа ди Бруно (см. [26]) влекут

$$
\begin{aligned}
& m_{n}^{\mathrm{BH}}(t ; k, l)=\sum_{r=1}^{L} m_{k, r} \int_{0}^{t} m_{n}^{\mathrm{BH}}(t-u ; r, l) d G_{k}(u) \\
& \quad+\left.\sum^{\sim} \frac{n !}{\prod_{p=1}^{n-1} i_{p}^{(1)} ! \cdots i_{p}^{(L)} !} \frac{\partial^{J(1)+\cdots+J(L)} g_{k}(\mathbf{s})}{\partial s_{1}^{J(1)} \cdots \partial s_{L}^{J(L)}}\right|_{\mathbf{s}=(1, \ldots, 1)} \\
& \quad \times \int_{0}^{t} \prod_{p=1}^{n-1}\left(\left(\frac{m_{p}^{\mathrm{BH}}(t-u ; 1, l)}{p !}\right)^{i_{p}^{(1)}} \cdots\left(\frac{m_{p}^{\mathrm{BH}}(t-u ; L, l)}{p !}\right)^{i_{p}^{(L)}}\right) d G_{k}(u) .
\end{aligned}
$$

Здесь символ $\tilde{\sum}$ означает сумму, взятую по всем $L$-мерным векторам $\mathbf{i}_{1}=\left(i_{1}^{(1)}, \ldots, i_{1}^{(L)}\right), \ldots, \mathbf{i}_{n-1}=\left(i_{n-1}^{(1)}, \ldots, i_{n-1}^{(L)}\right)$ с неотрицательными целыми компонентами, удовлетворяющими равенству $\sum_{p=1}^{n-1} p\left(i_{p}^{(1)}+\cdots+\right.$ $\left.i_{p}^{(L)}\right)=n$. Для каждого $r=1, \ldots, L$ также положим $J(r):=\sum_{p=1}^{n-1} i_{p}^{(r)}$. Подставляя в (32) явные формулы (1) и (2) для производящих функций числа потомков $g_{k}(\cdot), k=1, \ldots, L$, процесса Беллмана-Харриса, приходим к более компактному соотношению

$$
m_{n}^{\mathrm{BH}}(t ; k, l)=\sum_{r=1}^{L} m_{k, r} \int_{0}^{t} m_{n}^{\mathrm{BH}}(t-u ; r, l) d G_{k}(u)
$$




$$
\begin{aligned}
& +\mathbf{I}(k \in\{1, \ldots, N\}) \sum^{\approx} \frac{\alpha_{k} f_{k}^{(J)}(1) n !}{i_{1} ! \cdots i_{n-1} !} \\
& \times \int_{0}^{t} \prod_{p=1}^{n-1}\left(\frac{m_{p}^{\mathrm{BH}}(t-u ; k, l)}{p !}\right)^{i_{p}} d G_{k}(u),
\end{aligned}
$$

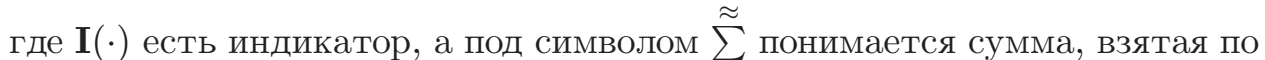
всем неотрицательным целым $i_{1}, \ldots, i_{n-1}$ таким, что $\sum_{p=1}^{n-1} p i_{p}=n$. Мы также используем обозначение $J:=i_{1}+\cdots+i_{n-1}$.

Ш а г 2. Теперь докажем часть утверждения (25), относящуюся к случаю $x, y \in W$. Итак, предположим, что $\rho(D)>1$. Применяя теорему 2.1, пункт (iii), из [19] к системе уравнений восстановления (31), получаем

$$
e^{-\nu t} m_{1}^{\mathrm{BH}}(t ; i, j) \rightarrow \frac{(-1)^{i+j} \operatorname{det}(I-H(\nu))_{j, i}}{\left.\left(\nu+\beta_{j}\right) \frac{d}{d \lambda} \operatorname{det}(I-H(\lambda))\right|_{\lambda=\nu}}, \quad t \rightarrow \infty
$$

для всех $i, j=1, \ldots, N$. Здесь $\nu>0$ и $\left.\frac{d}{d \lambda} \operatorname{det}(I-H(\lambda))\right|_{\lambda=\nu}>0$ (см., например, [27]). Сравнивая формулы (7) и (25) с (34), видим, что остается установить соотношения между определителями и алгебраическими дополнениями матриц $I-H(\lambda)$ и $I-D(\lambda)$. Следующая лемма дает эти соотношения.

Лемма 2. Для всех $\lambda \geqslant 0 u i=1, \ldots,\left|K_{j}\right|, j, k=1, \ldots, N$ имеем

$$
\begin{gathered}
\operatorname{det}(I-H(\lambda))=\operatorname{det}(I-D(\lambda)), \quad \operatorname{det}(I-H(\lambda))_{j, k}=\operatorname{det}(I-D(\lambda))_{j, k}, \\
(-1)^{L(j)+i+k} \operatorname{det}(I-H(\lambda))_{L(j)+i, k}=(-1)^{j+k} m_{j, L(j)+i} G_{j}^{*}(\lambda) \operatorname{det}(I-D(\lambda))_{j, k} .
\end{gathered}
$$

Д о к а з а т е л ь с т в о. Применим к столбцам матрицы $I-H(\lambda)$ преобразования, которые не изменят ее определителя. А именно, для всех $j, k=1, \ldots, N$ прибавим $(L(j)+i)$-й столбец, умноженный на $G_{L(j)+i}^{*}(\lambda)$, к $k$-му столбцу, если $k \in K_{j}$, т.е. $k=k(j, i)$ для некоторого $i=1, \ldots,\left|K_{j}\right|$. После этих преобразований мы получим блочную матрицу, состоящую из четырех блоков. Левый верхний блок размера $N \times N$ есть матрица $I-D(\lambda)$, в то время как левый нижний блок размера $(L-N) \times N$ и правый нижний блок размера $(L-N) \times(L-N)-$ это соответственно нулевая и единичная матрицы. Используя формулу для определителя блочной матрицы (см., например, [6, гл. 2, § 5]), приходим к первому утверждению леммы 2. Второе утверждение устанавливается аналогично. Для доказательства третьего утверждения применим описанные выше преобразования к матрице $(I-H(\lambda))_{L(j)+i, k}$ и затем переставим столбец под номером $L(j)+i-1$ на место столбца под номером $N$. Определители матрицы $(I-H(\lambda))_{L(j)+i, k}$ и преобразованной матрицы совпадают с точностью до множителя $(-1)^{L(j)+i-N-1}$. Далее 
мы пользуемся формулой для определителя блочной матрицы еще раз и применяем разложение определителя по $N$-му столбцу. Лемма 2 полностью доказана.

Таким образом, в силу (34) и леммы 2 мы доказали (25) при $n=1$ и $x, y \in W$. Для проверки соотношения (25) при $n>1$ и $x, y \in W$ используем индукцию по переменной $n$. Случай $n=1$ разобран выше. Пусть формула (25) верна для всех моментов порядков, не превышающих $n-1$. Тогда второй член в правой части $(33)$, обозначаемый $V_{n}(t ; k, l)$, имеет следующее асимптотическое поведение:

$V_{n}(t ; k, l) \sim \mathbf{I}(k \in\{1, \ldots, N\}) e^{n \nu t} G_{k}^{*}(n \nu) \stackrel{\approx}{\sum} \frac{\alpha_{k} f_{k}^{(J)}(1) n !}{i_{1} ! \cdots i_{n-1} !} \prod_{p=1}^{n-1}\left(\frac{a_{p}\left(w_{k}, w_{l}\right)}{p !}\right)^{i_{p}}$

при $t \rightarrow \infty$ для всех $k=1, \ldots, L$ и $l=1, \ldots, N$. Учитывая последнее соотношение и применяя теорему 2.1, пункт (iv), из [19] к системе уравнений восстановления (33), получаем

$$
\begin{aligned}
\frac{m_{n}^{\mathrm{BH}}(t ; i, j)}{e^{n \nu t}} \rightarrow & \sum_{k=1}^{N} \frac{(-1)^{i+k} \beta_{k} \operatorname{det}(I-H(n \nu))_{k, i}}{\left(n \nu+\beta_{k}\right) \operatorname{det}(I-H(n \nu))} \\
& \times h_{n, k}\left(a_{1}\left(w_{k}, w_{j}\right), \ldots, a_{n-1}\left(w_{k}, w_{j}\right)\right)
\end{aligned}
$$

при $t \rightarrow \infty$ для всех $i, j=1, \ldots, N$. Здесь мы пользуемся альтернативным представлением (см., например, [11, теорема 3.3]) для функции $h_{n, k}$, определенной в (10). Последнее асимптотическое соотношение в сочетании с леммой 2 и формулой (9) приводит к желаемому утверждению в $(25)$, когда $n>1$ и $x, y \in W$. Отметим, что функции $a_{n}\left(w_{i}, w_{j}\right), n \geqslant 1$, $i, j=1, \ldots, N$, строго положительны согласно, например, [6, гл. 13, § 3].

Ш а г 3. Далее мы докажем утверждение (27) при $x, y \in W$. Предположим, что $\rho(D)=1$ или, что эквивалентно, что единственное решение уравнения $\rho(D(\nu))=1$ есть $\nu=0$. Применяя теорему 2.1 , пункт (iii), из [19] к (31), приходим к следующему соотношению:

$$
m_{1}^{\mathrm{BH}}(t ; i, j) \rightarrow \frac{(-1)^{i+j} \operatorname{det}(I-H(0))_{j, i}}{\left.\beta_{j} \frac{d}{d \lambda} \operatorname{det}(I-H(\lambda))\right|_{\lambda=0+}}, \quad t \rightarrow \infty, \quad i, j=1, \ldots, N .
$$

Эта формула вместе с (15) и леммой 2 влечет за собой требуемое утверждение (27) при $n=1$. Для $n>1$ воспользуемся методом индукции по переменной $n$. Совершая шаг индукции, получаем

$$
\begin{aligned}
V_{n}(t ; k, l) & \sim \sum \frac{\alpha_{k} f_{k}^{(J)}(1) n !}{i_{1} ! \cdots i_{n-1} !} \prod_{p=1}^{n-1}\left(\frac{b_{p}\left(w_{k}, w_{l}\right)}{p !}\right)^{i_{p}} \int_{0}^{t}(t-u)^{n-J} d G_{k}(u) \\
& \sim t^{n-2} \frac{\alpha_{k} f_{k}^{\prime \prime}(1)}{2} \sum_{r=1}^{n-1}\left(\begin{array}{l}
n \\
r
\end{array}\right) b_{r}\left(w_{k}, w_{l}\right) b_{n-r}\left(w_{k}, w_{l}\right), \quad t \rightarrow \infty
\end{aligned}
$$


для $k, l=1, \ldots, N$, в то время как $V_{n}(t ; k, l)=0$ для $k=N+1, \ldots, L$, $l=1, \ldots, N$. Следовательно, применяя к (33) теорему 2.1, пункт (v), из [19], мы видим, что

$$
\begin{aligned}
\frac{m_{n}^{\mathrm{BH}}(t ; i, j)}{t^{n-1}} \rightarrow & \sum_{k=1}^{N} \frac{(-1)^{i+k} \alpha_{k} f_{k}^{\prime \prime}(1) \operatorname{det}(I-H(0))_{k, i}}{\left.2(n-1) \frac{d}{d \lambda} \operatorname{det}(I-H(\lambda))\right|_{\lambda=0+}} \\
& \times \sum_{r=1}^{n-1}\left(\begin{array}{l}
n \\
r
\end{array}\right) b_{r}\left(w_{k}, w_{j}\right) b_{n-r}\left(w_{k}, w_{j}\right)
\end{aligned}
$$

при $t \rightarrow \infty$ для всех $i, j=1, \ldots, N$. Утверждение (27) при $n>1$ теперь следует из последнего соотношения, определения (16) и леммы 2 . В силу $[6$, гл. $13, \S 3]$, алгебраическое дополнение $\Delta_{i, j}(0)$ строго положительно для любых $i, j=1, \ldots, N$. Как установлено в [27], $\Delta^{\prime}(0) \in(0, \infty]$. Более того, $\Delta^{\prime}(0)<\infty$ тогда и только тогда, когда $\int_{0}^{\infty} u d_{W_{j}} \bar{F}_{w_{i}, w_{j}}(u)<\infty$ для всех $i, j=1, \ldots, N$. Тем самым, функции $b_{n}\left(w_{i}, w_{j}\right)$ строго положительны при всех $n \in \mathbf{N}, i, j=1, \ldots, N$ тогда и только тогда, когда $\int_{0}^{\infty} u d_{W_{j}} \bar{F}_{w_{i}, w_{j}}(u)<\infty$ и, дополнительно, в случае возвратной цепи $\eta$ и $n \geqslant 2$ верно неравенство $\sum_{i=1}^{N} \alpha_{i}\left|f_{i}(s)-s\right|>0$ для некоторого $s \in[0,1)$. Заметим, что последнее условие позволяет выделить случай только перемещения частицы без размножения. Такое КВП является критическим для возвратной марковской цепи $\eta$ и докритическим для невозвратной $\eta$.

Ш а г 4. Соотношение (29) для случая $x, y \in W$ проверяется применением к (31) и (33) теоремы 2.2, пункт (ii), из [28]. Здесь мы существенно опираемся на тот факт, что $M^{n}$ сходится поэлементно к нулевой матрице при $n \rightarrow \infty$, поскольку в докритическом случае в силу леммы 1 выполнено неравенство $\rho(M)<1$.

Ш а г 5. Выведем систему уравнений восстановления, вовлекающую факториальные моменты общего числа частиц, когда $x \in W$ и цепь $\eta$ возвратна. При $t \geqslant 0$ обозначим $Z(t):=\sum_{j=1}^{L} Z_{j}(t)$ общее число частиц в момент $t$ во вспомогательном процессе Беллмана-Харриса. В соответствии с построением этого процесса законы распределения для $\mu(t)$ и $Z(t)$ совпадают при каждом $t \geqslant 0$ тогда и только тогда, когда марковская цепь $\eta$ возвратна. В самом деле, если марковская цепь $\eta$ невозвратна, то в момент $t$ в КВП с положительной вероятностью существуют частицы, имеющие бесконечные продолжительности жизни, поскольку они никогда не достигнут множества $W$ после момента $t$. Эти частицы не охватываются нашим $L$-мерным процессом БеллманаХарриса. Поэтому пока сосредоточимся на возвратном случае. Тогда имеем $M_{n}\left(t ; w_{i}\right)=M_{n}^{\mathrm{BH}}(t ; i)$ для всех $t \geqslant 0, n \in \mathbf{N}$ и $i=1, \ldots, N$, где $M_{n}^{\mathrm{BH}}(t ; k):=\mathbf{E}_{k} Z(t)(Z(t)-1) \cdots(Z(t)-n+1), t \geqslant 0, n \in \mathbf{N}$ и $k=1, \ldots, L$. Следовательно, при условии $\mathbf{E} \xi_{l}^{n}<\infty$ для некоторого $n \in \mathbf{N}$ и любых $l=1, \ldots, N$ теорема 1 из $[8$, гл. 8, $\S 6]$ влечет конечность функций $M_{n}\left(t ; w_{i}\right)$ для каждого $t \geqslant 0$ и всех $i=1, \ldots, N$. Более того, в силу этой 
теоремы функции $M_{1}^{\mathrm{BH}}(t ; k), t \geqslant 0, k=1, \ldots, L$, удовлетворяют следующей системе уравнений восстановления:

$$
M_{1}^{\mathrm{BH}}(t ; k)=1-G_{k}(t)+\sum_{r=1}^{L} m_{k, r} \int_{0}^{t} M_{1}^{\mathrm{BH}}(t-u ; r) d G_{k}(u) .
$$

При $n>1$ эта теорема и обобщенная формула Фаа ди Бруно (см. [26]) дают уравнения относительно $M_{n}^{\mathrm{BH}}(t ; k)$ такие же, как полученные из $(32)$ после замены $m_{n}^{\mathrm{BH}}(t ; k, l)$ на $M_{n}^{\mathrm{BH}}(t ; k)$. Тем самым, для $M_{n}^{\mathrm{BH}}(t ; k)$, $t \geqslant 0, k=1, \ldots, L$, система уравнений восстановления (33) имеет место, если функцию $m_{n}^{\mathrm{BH}}(t ; k, l)$ заменить на $M_{n}^{\mathrm{BH}}(t ; k)$.

Ш а г 6. Теперь рассмотрим КВП в случае, когда движение частиц управляется невозвратной марковской цепью $\eta$. Чтобы охватить невозвратный случай, построим новый вспомогательный процесс БеллманаХарриса с $L+1$ типами частиц, причем $(L+1)$-й тип является финальным (см., например, [3] и [8, гл. 5, § 3]). Для нового процесса БеллманаХарриса обозначим $\widehat{G}_{j}(t), t \geqslant 0$, функцию распределения продолжительности жизни частицы $j$-го типа, а $\widehat{g}_{j}\left(\mathbf{s}, s_{L+1}\right), \mathbf{s} \in[0,1]^{L}, s_{L+1} \in[0,1]$, производящую функцию ее потомков, $j=1, \ldots, L+1$. Пусть $\widehat{G}_{i}(t)=G_{i}(t)$, $t \geqslant 0$, для $i=1, \ldots, L$ и и $\widehat{g}_{j}\left(\mathbf{s}, s_{L+1}\right)=g_{j}(\mathbf{s}), \mathbf{s} \in[0,1]^{L}, s_{L+1} \in[0,1]$, для $j=N+1, \ldots, L$, где $G_{i}$ и $g_{j}$ определены в (1) и (2). Положим

$$
\begin{aligned}
\widehat{g}_{j}\left(\mathbf{s}, s_{L+1}\right)= & \alpha_{j} f_{j}\left(s_{j}\right)+\left(1-\alpha_{j}\right) \sum_{k=1}^{N} W_{k} \bar{F}_{w_{j}, w_{k}}(0) s_{k} \\
& +\left(1-\alpha_{j}\right) \sum_{i=1}^{\left|K_{j}\right|} T_{i, j}(\infty) s_{L(j)+i} \\
& +\left(1-\alpha_{j}\right)\left(1-\sum_{k=1}^{N} W_{k} \bar{F}_{w_{j}, w_{k}}(\infty)\right) s_{L+1}
\end{aligned}
$$

при $j=1, \ldots, N, \mathbf{s} \in[0,1]^{L}, s_{L+1} \in[0,1]$, т.е. определения функций $g_{j}$ и $\widehat{g}_{j}$ отличаются только последним слагаемым. Поскольку $(L+1)$-й тип является финальным (другими словами, каждая частица типа $L+1$ обладает бесконечной продолжительностью жизни и не производит потомков), для определенности мы можем положить $\widehat{G}_{L+1}(t)=0, t \geqslant 0$, и $\widehat{g}_{L+1}\left(\mathbf{s}, s_{L+1}\right)=$ $s_{L+1}, \mathbf{s} \in[0,1]^{L}, s_{L+1} \in[0,1]$. Матрица средних числа непосредственных потомков частиц различных типов нового процесса имеет элементы $\widehat{m}_{k, l}=m_{k, l}, k, l=1, \ldots, L, \widehat{m}_{i, L+1}=\left(1-\alpha_{i}\right)\left(1-\sum_{k=1}^{N} W_{k} \bar{F}_{w_{i}, w_{k}}(\infty)\right)$, $i=1, \ldots, N, \widehat{m}_{L+1, L+1}=1$ и $\widehat{m}_{k, l}=0$ для остальных пар $k$ и $l$. Пусть $\widehat{Z}_{j}(t), t \geqslant 0, j=1, \ldots, L+1$, есть число частиц типа $j$ в момент $t$ в новом процессе Беллмана-Харриса. Очевидно, распределения векторов $\left(\widehat{Z}_{1}(t), \ldots, \widehat{Z}_{L}(t)\right)$ и $\left(Z_{1}(t), \ldots, Z_{L}(t)\right)$ совпадают для каждого $t \geqslant 0$, если родительские частицы обоих процессов Беллмана-Харриса принадлежат одному и тому же типу. Более того, новый процесс учитывает даже 
частицы из $(L+1)$-й группы частиц в КВП (см. раздел 2$)$, которые не охватываются $L$-мерным процессом Беллмана-Харриса. Таким образом, для всех $n \in \mathbf{N}, i=1, \ldots, N$ и $t \geqslant 0$ имеем $M_{n}\left(t ; w_{i}\right)=\widehat{M}_{n}^{\mathrm{BH}}(t ; i)$, где $\widehat{M}_{n}^{\mathrm{BH}}(t ; k):=\mathbf{E}_{k} \widehat{Z}(t)(\widehat{Z}(t)-1) \cdots(\widehat{Z}(t)-n+1), k=1, \ldots, L+1$, и $\widehat{Z}(t):=\sum_{j=1}^{L+1} \widehat{Z}_{j}(t)$. Применяя теорему 1 из [8, гл. $\left.8, \S 6\right]$, мы убеждаемся в том, что если $\mathbf{E} \xi_{l}^{n}<\infty$ для некоторого $n \in \mathbf{N}$ и любых $l=1, \ldots, N$, то функции $M_{n}\left(t ; w_{i}\right)$ конечны для каждого $t \geqslant 0$ и всех $i=1, \ldots, N$. Эта теорема также влечет, что функции $\widehat{M}_{1}^{\mathrm{BH}}(t ; k), t \geqslant 0, k=1, \ldots, L+1$, удовлетворяют следующей системе из $L+1$ уравнения восстановления:

$$
\widehat{M}_{1}^{\mathrm{BH}}(t ; k)=1-\widehat{G}_{k}(t)+\sum_{r=1}^{L+1} \widehat{m}_{k, r} \int_{0}^{t} \widehat{M}_{1}^{\mathrm{BH}}(t-u ; r) d \widehat{G}_{k}(u)
$$

Уравнение под номером $L+1$ есть просто $\widehat{M}_{1}^{\mathrm{BH}}(t ; L+1)=1, t \geqslant 0$. Подставляя это значение 1 и выражения для $\widehat{m}_{k, r}, r=1, \ldots, L+1$, в первые $L$ уравнений, приходим к новой системе из $L$ уравнений восстановления:

$$
\begin{aligned}
\widehat{M}_{1}^{\mathrm{BH}}(t ; k)= & \sum_{r=1}^{L} m_{k, r} \int_{0}^{t} \widehat{M}_{1}^{\mathrm{BH}}(t-u ; r) d G_{k}(u)+1-G_{k}(t) \\
& +\mathbf{I}(k \in\{1, \ldots, N\})\left(1-\alpha_{k}\right) \\
& \times\left(1-\sum_{j=1}^{N} W_{j} \bar{F}_{w_{k}, w_{j}}(\infty)\right) G_{k}(t)
\end{aligned}
$$

при $t \geqslant 0$ и $k=1, \ldots, L$. Нетрудно проверить, что в силу теоремы 1 из $[8$, гл. $8, \S 6]$, система из $L+1$ уравнения, получающаяся из $(32)$ в результате замены $L$ на $L+1$ и $m_{n}^{\mathrm{BH}}(t ; k, l)$ на $\widehat{M}_{n}^{\mathrm{BH}}(t ; k)$, справедлива при $n>1, t \geqslant 0$ и $k=1, \ldots, L+1$. Уравнение под номером $L+1$ есть просто $\widehat{M}_{n}^{\mathrm{BH}}(t ; L+1)=0$. Подставляя его в первые $L$ уравнений и упрощая их аналогично выводу (33), приходим к системе из $L$ уравнений восстановления относительно функций $\widehat{M}_{n}^{\mathrm{BH}}(t ; k)$ при $n>1, t \geqslant 0$ и $k=1, \ldots, L$ такой же, как $(33)$, но с $\widehat{M}_{n}^{\text {вн }}(t ; k)$ вместо $m_{n}^{\text {ВН }}(t ; k, l)$.

Ш а г 7. Теперь докажем утверждение (26) для $x, y \in W$. Итак, предположим, что $\rho(D)>1$. Сначала рассмотрим возвратный случай. Применяя теорему 2.1, пункт (iii), из [19] к (35), мы видим, что

$$
\begin{gathered}
\frac{M_{1}^{\mathrm{BH}}(t ; i)}{e^{\nu t}} \rightarrow \sum_{j=1}^{N} \frac{\Delta_{j, i}(\nu)}{\nu \Delta^{\prime}(\nu)}\left(1-\alpha_{j} G_{j}^{*}(\nu)-\left(1-\alpha_{j}\right) G_{j}^{*}(\nu) \sum_{k=1}^{N} W_{k} \bar{F}_{w_{j}, w_{k}}^{*}(\nu)\right) \\
=\sum_{k=1}^{N} \sum_{j=1}^{N} \frac{\Delta_{j, i}(\nu)}{\nu \Delta^{\prime}(\nu)}\left(\delta_{j, k}-\delta_{j, k} \alpha_{j} f_{j}^{\prime}(1) G_{j}^{*}(\nu)-\left(1-\alpha_{j}\right) G_{j}^{*}(\nu)_{W_{k}} \bar{F}_{w_{j}, w_{k}}^{*}(\nu)\right) \\
\quad+\sum_{j=1}^{N} \frac{\alpha_{j}\left(f_{j}^{\prime}(1)-1\right) G_{j}^{*}(\nu) \Delta_{j, i}(\nu)}{\nu \Delta^{\prime}(\nu)}=\sum_{j=1}^{N} \frac{\alpha_{j} \beta_{j}\left(f_{j}^{\prime}(1)-1\right) \Delta_{j, i}(\nu)}{\nu\left(\nu+\beta_{j}\right) \Delta^{\prime}(\nu)}
\end{gathered}
$$


при $t \rightarrow \infty$ и $i=1, \ldots, N$. Здесь мы опираемся на лемму 2 и формулы (1)-(3), а также на разложение определителя по столбцу и очевидное равенство $\sum_{k=1}^{N} W_{k} \bar{F}_{w_{j}, w_{k}}(\infty)=1$, справедливое в возвратном случае. Мы также учли, что $\Delta(\nu)=0$, поскольку 1 является собственным значением матрицы $D(\nu)$. Соотношение (37) наряду с (12) приводит к желаемому утверждению (26), когда $n=1$ и марковская цепь $\eta$ возвратна. Невозвратный случай разбирается аналогично за исключением двух отличий. Первое состоит в применении системы уравнений (36) вместо (35). Второе отличие заключается в том, что для невозвратной марковской цепи $\eta$ строгое неравенство $\sum_{k=1}^{N} W_{k} \bar{F}_{w_{j}, w_{k}}(\infty)<1$ имеет место хотя бы для одного $j \in\{1, \ldots, N\}$. Однако при выводе соотношения для $\widehat{M}_{1}^{\mathrm{BH}}(t ; i)$, аналогичного $(37)$, разница между последней суммой и 1 компенсируется за счет дополнительного слагаемого в (36). Таким образом, при $n=1$ утверждение (26) установлено и асимптотическое поведение функций $M_{1}\left(t ; w_{i}\right), i=1, \ldots, N$, в надкритическом КВП не зависит от того, является марковская цепь $\eta$ возвратной или нет. Поскольку при $n>1$ системы уравнений относительно соответственно $M_{n}^{\mathrm{BH}}(t ; k)$ и $\widehat{M}_{n}^{\text {вН }}(t ; k)$ имеют такой же вид, как $(33)$, асимптотическое поведение функции $M_{n}\left(t ; w_{i}\right)$ в надкритическом КВП также не зависит от возвратности или невозвратности $\eta$. Более того, при $n>1$ вывод формулы (26) для общего числа частиц почти дословно повторяет вывод (29) для локальных численностей частиц и по этой причине опускается. Заметим, что положительность функции $A_{1}\left(w_{i}\right)$ (а следовательно, и $A_{n}\left(w_{i}\right)$ при $n>1$ ) для каждого $i=1, \ldots, N$ следует из уже доказанного соотношения (25).

Ш а г 8. Далее перейдем к доказательству утверждения (28) для $x, y \in W$. Итак, рассмотрим случай $\rho(D)=1$. Применяя теорему 2.1, пункт (v), из [19] к (36), получаем

$$
\begin{aligned}
t^{-1} & \widehat{M}_{1}^{\mathrm{BH}}(t ; i) \rightarrow \sum_{j=1}^{N} \frac{\left(1-\alpha_{j}\right) \Delta_{j, i}(0)}{\Delta^{\prime}(0)}\left(1-\sum_{k=1}^{N} W_{k} \bar{F}_{w_{j}, w_{k}}(\infty)\right) \\
= & \sum_{k=1}^{N} \sum_{j=1}^{N} \frac{\Delta_{j, i}(0)}{\Delta^{\prime}(0)}\left(\delta_{j, k}-\delta_{j, k} \alpha_{j} f_{j}^{\prime}(1)-\left(1-\alpha_{j}\right)_{W_{k}} \bar{F}_{w_{j}, w_{k}}(\infty)\right) \\
& +\sum_{j=1}^{N} \frac{\alpha_{j}\left(f_{j}^{\prime}(1)-1\right) \Delta_{j, i}(0)}{\Delta^{\prime}(0)} \\
= & \sum_{j=1}^{N} \frac{\alpha_{j}\left(f_{j}^{\prime}(1)-1\right) \Delta_{j, i}(0)}{\Delta^{\prime}(0)}, \quad t \rightarrow \infty
\end{aligned}
$$

при $i=1, \ldots, N$. Здесь мы используем лемму 2, а также разложение определителя по столбцу и равенство $\Delta(0)=0$, справедливое в силу предположения $\rho(D)=1$. Соотношение (38) в сочетании с (18) влечет 
требуемое утверждение в (28), когда $n=1$ и марковская цепь $\eta$ невозвратна. Если марковская цепь $\eta$ возвратна, соответствующее утверждение вытекает из [19, теорема 2.1, пункт (i)] и наблюдения, заключающегося в том, что неинтегральный член в правой части (35) стремится к 0 при $t \rightarrow \infty$, в то время как в (36) он сходится к положительному пределу хотя бы для одного $k \in\{1, \ldots, N\}$. Для $n>1$ вывод формулы (28) для общего числа частиц снова воспроизводит вывод (27) для локальных численностей частиц и по этой причине опускается. Отметим, что в силу (38) функция $B_{1}\left(w_{i}\right)$ (а следовательно, и $B_{n}\left(w_{i}\right)$ при $n>1)$ строго положительна для каждого $i=1, \ldots, N$ тогда и только тогда, когда $\Delta^{\prime}(0)<\infty$ и $\sum_{k=1}^{N} W_{k} \bar{F}_{w_{j}, w_{k}}(\infty)<1$ по крайней мере для одного $j \in\{1, \ldots, N\}$.

Ш а г 9. Наконец, установим (30) для случая $x, y \in W$. Предположим, что $\rho(D)<1$. Применяя теорему 2.2 , пункт (ii), из [28] к $(36)$, мы видим, что

$$
\begin{aligned}
& \widehat{M}_{1}^{B H}(t ; i) \rightarrow \sum_{j=1}^{N} \frac{\left(1-\alpha_{j}\right) \Delta_{j, i}(0)}{\Delta(0)}\left(1-\sum_{k=1}^{N} W_{k} \bar{F}_{w_{j}, w_{k}}(\infty)\right) \\
& =\sum_{k=1}^{N} \sum_{j=1}^{N} \frac{\Delta_{j, i}(0)}{\Delta(0)}\left(\delta_{j, k}-\delta_{j, k} \alpha_{j} f_{j}^{\prime}(1)-\left(1-\alpha_{j}\right)_{W_{k}} \bar{F}_{w_{j}, w_{k}}(\infty)\right) \\
& \quad+\sum_{j=1}^{N} \frac{\alpha_{j}\left(f_{j}^{\prime}(1)-1\right) \Delta_{j, i}(0)}{\Delta(0)} \\
& =1+\sum_{j=1}^{N} \frac{\alpha_{j}\left(f_{j}^{\prime}(1)-1\right) \Delta_{j, i}(0)}{\Delta(0)}, \quad t \rightarrow \infty
\end{aligned}
$$

для каждого $i=1, \ldots, N$. Здесь мы учитываем лемму 2 , а также разложение определителя по столбцу и неравенство $\Delta(0)>0$, верное в силу предположения $\rho(D)<1$. Формулы $(21)$ и $(39)$ влекут желаемое утверждение (30), когда $n=1$ и марковская цепь $\eta$ невозвратна. Если марковская цепь $\eta$ возвратна, то соответствующее соотношение следует из (35) и теоремы 2.2, пункт (ii), из [28]. Когда $n>1$, эта теорема также приводит к (30) ввиду системы уравнений (33). Заметим, что в силу (39) функция $C_{1}\left(w_{i}\right)$ (а следовательно, и $C_{n}\left(w_{i}\right)$ при $n>1$ ) строго положительна для каждого $i=1, \ldots, N$ тогда и только тогда, когда $\sum_{k=1}^{N} W_{k} \bar{F}_{w_{j}, w_{k}}(\infty)<1$ хотя бы для одного $j \in\{1, \ldots, N\}$, т.е. когда марковская цепь $\eta$ невозвратна.

Таким образом, теорема 1 полностью доказана для случая $x, y \in W$.

Случай 2. Теперь мы предполагаем, что либо $x \in S \backslash W$, либо $y \in S \backslash W$, либо $x, y \in S \backslash W$. Основная идея оставшейся части доказательства состоит в следующем. Если $x \in S \backslash W$ и $y \in W$, то мы пополняем множество катализаторов $W$ элементом $x$. Наоборот, если 
$x \in W$, а $y \in S \backslash W$, то дополним множество катализаторов $W$ элементом $y$. Если $x \in S \backslash W$ и $y=x$, то добавим $x$ к множеству катализаторов $W$. Если же и $x$, и $y$ принадлежат $S \backslash W$ и, более того, $x \neq y$, то дополним множество катализаторов $W$ двумя состояниями $x$ и $y$. Далее мы можем использовать уже установленные результаты для КВП с $N+1$ или $N+2$ катализаторами. Итак, положим $w_{N+1}=x, W(x):=W \cup\{x\}$ и $W_{i}(x):=W(x) \backslash\left\{w_{i}\right\}, i=1, \ldots, N+1$. Пусть $D(x ; \lambda)=\left(d_{i, j}(x ; \lambda)\right)_{i, j=1}^{N+1}$ есть матрица с элементами

$$
d_{i, j}(x ; \lambda):=\delta_{i, j} \alpha_{i} f_{i}^{\prime}(1) G_{i}^{*}(\lambda)+\left(1-\alpha_{i}\right) G_{i}^{*}(\lambda)_{W_{j}(x)} \bar{F}_{w_{i}, w_{j}}^{*}(\lambda), \quad \lambda \geqslant 0 .
$$

Здесь $\alpha_{N+1}=0, f_{N+1}^{\prime}(1)=0$ и $G_{N+1}(t):=1-e^{q(x, x) t}, t \geqslant 0$. Аналогично, положим $w_{N+2}=y, W(x, y):=W(x) \cup\{y\}$ и $W_{i}(x, y):=W(x, y) \backslash\left\{w_{i}\right\}$, $i=1, \ldots, N+2$. Рассмотрим матрицу $D(x, y ; \lambda)=\left(d_{i, j}(x, y ; \lambda)\right)_{i, j=1}^{N+2} \mathrm{c}$ элементами

$$
d_{i, j}(x, y ; \lambda):=\delta_{i, j} \alpha_{i} f_{i}^{\prime}(1) G_{i}^{*}(\lambda)+\left(1-\alpha_{i}\right) G_{i}^{*}(\lambda)_{W_{j}(x, y)} \bar{F}_{w_{i}, w_{j}}^{*}(\lambda)
$$

при $\lambda \geqslant 0$. Здесь $\alpha_{N+2}=0, f_{N+2}^{\prime}(1)=0$ и $G_{N+2}(t):=1-e^{q(y, y) t}, t \geqslant 0$. Наконец, обозначим $\nu(x)$ и $\nu(x, y)$ единственные решения соответственно уравнений $\rho(D(x ; \nu(x)))=1$ и $\rho(D(x, y ; \nu(x, y)))=1$. Следующая лемма, доказательство которой аналогично доказательству леммы 1 и поэтому опускается, показывает, что $\nu=\nu(x)=\nu(x, y)$.

Лемма 3. Для каждого $\lambda \geqslant 0$ перроновы корни $\rho(D(\lambda)), \rho(D(x ; \lambda))$ и $\rho(D(x, y ; \lambda))$ одновременно больще 1, равны 1 или меньше 1.

После применения установленной части теоремы 1 к КВП с множеством катализаторов $W(x)$ или $W(x, y)$ замечаем, что для завершения доказательства теоремы 1 требуется привести выражения вида $a_{n}\left(w_{N+1}, w_{N+2}\right)$ к форме (11). Это может быть легко проделано с помощью следующего результата, доказательство которого проводится в том же ключе, что и доказательство леммы 2.

Лемма 4. Для $x, y \in S \backslash W, x \neq y, i, j=1, \ldots, N u \lambda \geqslant 0$ справедливы равенства

$$
\begin{gathered}
\operatorname{det}(I-D(x ; \lambda))=\Delta(\lambda)\left(1-{ }_{W} F_{x, x}^{*}(\lambda)\right) \\
\operatorname{det}(I-D(x, y ; \lambda))=\Delta(\lambda)\left(1-{ }_{W} F_{x, x}^{*}(\lambda)\right)\left(1-{ }_{W(x)} F_{y, y}^{*}(\lambda)\right) \\
\left.\frac{d}{d \lambda} \operatorname{det}(I-D(x ; \lambda))\right|_{\lambda=\nu}=\Delta^{\prime}(\nu)\left(1-{ }_{W} F_{x, x}^{*}(\nu)\right) \\
\left.\frac{d}{d \lambda} \operatorname{det}(I-D(x, y ; \lambda))\right|_{\lambda=\nu}=\Delta^{\prime}(\nu)\left(1-{ }_{W} F_{x, x}^{*}(\nu)\right)\left(1-{ }_{W(x)} F_{y, y}^{*}(\nu)\right), \\
(-1)^{i+j} \operatorname{det}(I-D(x ; \lambda))_{j, i}=\Delta_{j, i}(\lambda)\left(1-{ }_{W} F_{x, x}^{*}(\lambda)\right),
\end{gathered}
$$




$$
\begin{gathered}
\frac{(-1)^{j+N+1} \operatorname{det}(I-D(x ; \lambda))_{j, N+1}}{1-{ }_{W} F_{x, x}^{*}(\lambda)}=\sum_{i=1}^{N} W_{i} F_{x, w_{i}}^{*}(\lambda) \Delta_{j, i}(\lambda) \\
(-1)^{i+N+1} \operatorname{det}(I-D(x ; \lambda))_{N+1, i}=\sum_{j=1}^{N} \Delta_{j, i}(\lambda)\left(1-\alpha_{j}\right) G_{j}^{*}(\lambda)_{W} \bar{F}_{w_{j}, x}^{*}(\lambda), \\
\operatorname{det}(I-D(x ; \lambda))_{N+1, N+1} \\
=\Delta(\lambda)+\sum_{i, j=1}^{N}{ }_{W i} F_{x, w_{i}}^{*}(\lambda) \Delta_{j, i}(\lambda)\left(1-\alpha_{j}\right) G_{j}^{*}(\lambda)_{W} \bar{F}_{w_{j}, x}^{*}(\lambda), \\
\frac{\operatorname{det}(I-D(x, y ; \lambda))_{N+2, N+1}\left(1-{ }_{W} F_{y, y}^{*}(\lambda)\right)}{\left(1-{ }_{W} F_{x, x}^{*}(\lambda)\right)\left(1-{ }_{W(x)} F_{y, y}^{*}(\lambda)\right)} \\
=\Delta(\lambda)_{W} F_{x, y}^{*}(\lambda)+\sum_{i, j=1}^{N}{ }_{W_{i}} F_{x, w_{i}}^{*}(\lambda) \Delta_{j, i}(\lambda)\left(1-\alpha_{j}\right) G_{j}^{*}(\lambda)_{W} \bar{F}_{w_{j}, y}^{*}(\lambda),
\end{gathered}
$$

где $\nu \geqslant 0$ является единственным решением уравнения $\rho(D(\nu))=1$, существуюшим, когда $\rho(D) \geqslant 1$. Если $\nu=0,\left.\operatorname{mo} \frac{d}{d \lambda} \operatorname{det}(I-D(x ; \lambda))\right|_{\lambda=\nu}$ означает правую производную в 0.

Теорема 1 полностью доказана.

4. Применения. В разделе 3 с помощью теоремы 1 установлено, что мальтусовский параметр $\nu$ играет ключевую роль в асимптотическом поведении как общих, так и локальных численностей частиц в надкритическом КВП. Прежде чем рассмотреть некоторые частные случаи в настоящем разделе, обсудим альтернативный способ нахождения $\nu$. $\mathrm{C}$ этой целью введем матрицы $\widetilde{D}(\lambda)=\left(\widetilde{d}_{i, j}(\lambda)\right)_{i, j=1}^{N}$ и $\widehat{D}(\lambda)=\left(\widehat{d}_{i, j}(\lambda)\right)_{i, j=1}^{N}$ с соответствующими элементами

$$
\begin{aligned}
\widetilde{d}_{i, j}(\lambda):= & \left(\alpha_{i} f_{i}^{\prime}(1) G_{i}^{*}(\lambda)-1+\left(1-\alpha_{i}\right) G_{i}^{*}(\lambda) \frac{\lambda-q\left(w_{i}, w_{i}\right)}{-q\left(w_{i}, w_{i}\right)}\right) F_{w_{i}, w_{j}}^{*}(\lambda) \\
& +\delta_{i, j}\left(\alpha_{i} f_{i}^{\prime}(1) G_{i}^{*}(\lambda)-1\right)\left(1-F_{w_{i}, w_{i}}^{*}(\lambda)\right), \quad \lambda \geqslant 0, \\
\widehat{d}_{i, j}(\lambda):= & \left(\alpha_{i} f_{i}^{\prime}(1) G_{i}^{*}(\lambda)-1+\left(1-\alpha_{i}\right) G_{i}^{*}(\lambda) \frac{\lambda-q\left(w_{i}, w_{i}\right)}{-q\left(w_{i}, w_{i}\right)}\right) G_{\lambda}\left(w_{i}, w_{j}\right) \\
& +\delta_{i, j} \frac{1-\alpha_{i}}{q\left(w_{i}, w_{i}\right)} G_{i}^{*}(\lambda), \quad \lambda>0 .
\end{aligned}
$$

Здесь $G_{\lambda}(x, y):=\int_{0}^{\infty} e^{-\lambda t} p(t ; x, y) d t, \lambda>0$, есть преобразование Лапласа переходной вероятности $p(t ; x, y), x, y \in S, t \geqslant 0$, марковской цепи $\eta$. Заметим, что функция $G_{0}(x, y)=\lim _{\lambda \rightarrow 0+} G_{\lambda}(x, y)$ называется функиией Грина и является конечной тогда и только тогда, когда марковская цепь $\eta$ невозвратна (см., например, теорему 4 и следствие 2 в $[9$, гл. $2, \S 10]$ ). 
Напомним также, что согласно теореме 3 и соотношению (4) из $[9$, гл. 2 , $\S 12]$ имеем

$$
F_{w_{i}, w_{j}}^{*}(\lambda)=\frac{G_{\lambda}\left(w_{i}, w_{j}\right)}{G_{\lambda}\left(w_{j}, w_{j}\right)}, \quad F_{w_{i}, w_{i}}^{*}(\lambda)=1-\frac{1}{\left(\lambda-q\left(w_{i}, w_{i}\right)\right) G_{\lambda}\left(w_{i}, w_{i}\right)}
$$

для любых $i, j=1, \ldots, N, i \neq j$ и $\lambda>0$.

Из леммы 1 и определения мальтусовского параметра в разделе 3 следует, что параметр $\nu$ может быть найден как наибольшее число $\lambda$, удовлетворяющее соотношению $\operatorname{det}(D(\lambda)-I)=0$. Следующая лемма позволяет найти $\nu$ как наибольшее число $\lambda$, являющееся решением уравнения $\operatorname{det} \widetilde{D}(\lambda)=0$ или равносильного уравнения $\operatorname{det} \widehat{D}(\lambda)=0$. Заметим, что элементы матрицы $D(\lambda)$ выражаются через преобразования Лапласа времен достижения с запретами в множествах $W_{i}, i=1, \ldots, N$, в то время как элементы матрицы $\widetilde{D}(\lambda)$ представлены в терминах преобразований Лапласа времен достижения без запретов. Наконец, элементы матрицы $\widehat{D}(\lambda)$ вовлекают преобразования Лапласа только переходных вероятностей.

Лемма 5. Для любого $\lambda>0$ имеем $\operatorname{det}(D(\lambda)-I)=0$ тогда $и$ только тогда, когда $\operatorname{det} \widetilde{D}(\lambda)=0$ или, эквивалентно, когда $\operatorname{det} \widehat{D}(\lambda)=$ 0. Более того, эти соотношения справедливь даже при $\lambda=0$, если иепь $\eta$ невозвратна.

Д о к а з а т е л ь с в о. Введем матрицы $\widetilde{R}(\lambda)=\left(\widetilde{r}_{i, j}(\lambda)\right)_{i, j=1}^{N}$ и $\widehat{R}(\lambda)=\left(\widehat{r}_{i, j}(\lambda)\right)_{i, j=1}^{N}, \lambda>0$, с соответствующими элементами $\tilde{r}_{i, j}(\lambda):=$ $\delta_{i, j}+\left(1-\delta_{i, j}\right) F_{w_{i}, w_{j}}^{*}(\lambda)$ и $\widehat{r}_{i, j}(\lambda):=G_{\lambda}\left(w_{i}, w_{j}\right)$. Согласно формуле $(49)$ столбец $j=1, \ldots, N$ матрицы $\widehat{R}(\lambda)$ получается из $j$-го столбца матрицы $\widetilde{R}(\lambda)$ в результате умножения его на $G_{\lambda}\left(w_{j}, w_{j}\right)$, где $G_{\lambda}\left(w_{i}, w_{j}\right)>0$ в силу неразложимости $\eta$. Следовательно, $\operatorname{det} \widetilde{R}(\lambda)=0$ тогда и только тогда, когда $\operatorname{det} \widehat{R}(\lambda)=0$ для любого $\lambda>0$. Покажем, что $\operatorname{det} \widehat{R}(\lambda) \neq 0$, если $\lambda>0$. Аналогично доказательству теоремы 8 из [9, гл. $2, \S 11]$ можно вывести, что

$$
F_{w_{i}, w_{j}}(t)={ }_{W_{j}} F_{w_{i}, w_{j}}(t)+\sum_{k=1, k \neq j}^{N} \int_{0}^{t}{ }_{W_{k}} F_{w_{i}, w_{k}}(t-u) d F_{w_{k}, w_{j}}(u)
$$

для всех $i, j=1, \ldots, N$. Заметим, что последнее соотношение имеет естественную интерпретацию. А именно, путь марковской цепи из $w_{i}$ в $w_{j}$ может либо избежать множество $W_{j}$, либо пройти через некоторый элемент из $W_{j}$ и потом уже достичь $w_{j}$. Применяя преобразование Лапласа-Стилтьеса к (50), получаем

$$
F_{w_{i}, w_{j}}^{*}(\lambda)={ }_{W_{j}} F_{w_{i}, w_{j}}^{*}(\lambda)+\sum_{k=1, k \neq j}^{N} W_{k} F_{w_{i}, w_{k}}^{*}(\lambda) F_{w_{k}, w_{j}}^{*}(\lambda) .
$$


Умножая каждую часть равенства (51) на $G_{\lambda}\left(w_{j}, w_{j}\right)$ и учитывая (49), мы видим, что

$G_{\lambda}\left(w_{i}, w_{j}\right)-\frac{\delta_{i, j}}{\lambda-q\left(w_{i}, w_{i}\right)}=\sum_{k=1}^{N} W_{k} F_{w_{i}, w_{k}}^{*}(\lambda) G_{\lambda}\left(w_{k}, w_{j}\right), \quad i, j=1, \ldots, N$.

Перепишем эти тождества в следующем матричном виде:

$$
\widehat{R}(\lambda)-T(\lambda)=U(\lambda) \widehat{R}(\lambda) \quad \text { или, эквивалентно, } \widehat{R}(\lambda)(I-U(\lambda))=T(\lambda),
$$

где

$$
T(\lambda):=\left(\delta_{i, j}\left(\lambda-q\left(w_{i}, w_{i}\right)\right)^{-1}\right)_{i, j=1}^{N}, \quad U(\lambda):=\left(W_{j} F_{w_{i}, w_{j}}^{*}(\lambda)\right)_{i, j=1}^{N},
$$

$\lambda>0$. Отсюда заключаем, что $\operatorname{det} \widehat{R}(\lambda) \neq 0$ для каждого $\lambda>0$, поскольку определитель $\operatorname{det} T(\lambda)$ строго положителен.

Из $(51)$ следует, что $(D(\lambda)-I) \widetilde{R}(\lambda)=\widetilde{D}(\lambda)$. Рассматривая определители матриц в левой и правой частях последнего равенства, приходим к первому утверждению леммы 5. Ее второе утверждение вытекает из представления $\widetilde{d}_{i, j}(\lambda) G_{\lambda}\left(w_{j}, w_{j}\right)=\widehat{d}_{i, j}(\lambda)$, справедливого для всех $\lambda>0$ и $i, j=1, \ldots, N$ в силу (49).

Все вышеприведенные рассуждения остаются верными даже при $\lambda=0$, если марковская цепь $\eta$ невозвратна. Таким образом, лемма 5 полностью доказана.

Рассмотрим некоторые применения наших результатов к моделям, изучавшимся ранее разными исследователями.

П р и м е р 1. Сосредоточимся на каталитическом ветвящемся случайном блуждании по $\mathbf{Z}^{d}, d \in \mathbf{N}$, предложенном в [32]. Эта модель является частным случаем КВП, если мы допустим в качестве $\eta$ симметричное и пространственно-однородное случайное блуждание по $\mathbf{Z}^{d} \mathrm{c}$ конечной дисперсией скачков, а также положим $N=1, w_{1}=\mathbf{0} \in \mathbf{Z}^{d}$, $\alpha_{1}=\alpha, \beta_{1}=1$ и $f_{1}(\cdot)=f(\cdot)$. Тогда мы выводим то же условие критичности, которое использовалось в [4], т.е. $D=1$ или, эквивалентно, $\alpha f^{\prime}(1)+(1-\alpha)\left(1+q(\mathbf{0}, \mathbf{0})^{-1} G_{0}^{-1}(\mathbf{0}, \mathbf{0})\right)=1$. Для возвратной марковской цепи $\eta$ полагаем $G_{0}^{-1}(\mathbf{0}, \mathbf{0})$ равным 0 . Применяя теорему 1 к каталитическому ветвящемуся случайному блужданию по $\mathbf{Z}^{d}$, приходим к теореме 4.1 и теореме 4.2 из [10], а также некоторым утверждениям теоремы 5 из [4] и теоремы 1 из [16].

П р и м е р 2. Рассмотрим каталитический ветвящийся процесс с одним катализатором (расположенным, скажем, в некоторой точке $w \in S)$, изучавшийся в [21]. Здесь перемещение частиц управляется неразложимой марковской цепью $\eta$. Тем самым, такая постановка задачи менее ограничительна, чем в примере 1. Как показано в [21], асимптотическое поведение общих и локальных численностей частиц 
зависит от того, будет ли среднее число потомков, производимых частицей в месте нахождения катализатора, меньше, равно или больше $G_{0}^{-1}(w, w)+1$, а также от возвратности/невозвратности $\eta$. Мы приходим к той же классификации, полагая $N=1, w_{1}=w, \alpha_{1}=(1-q(w, w))^{-1}$, $\beta_{1}=1-q(w, w)$ и $f_{1}^{\prime}(1)=m$ в нашем КВП. Тогда в силу (49) и очевидной формулы $F_{w, w}(\infty)=\lim _{\lambda \rightarrow 0+} F_{w, w}^{*}(\lambda)$ значение $\operatorname{sign}(D-1)$ совпадает c $\operatorname{sign}\left(m-G_{0}^{-1}(w, w)-1\right)$. Подчеркнем, что в отличие от [21] мы не предполагаем существование всех моментов числа потомков, т.е. конечности $f_{1}^{(k)}(1)$ для любого $k \in \mathbf{N}$. Некоторые утверждения теоремы 1 в случае КВП с одним катализатором сильнее, чем соответствующие утверждения теоремы 1 из [21] (например, стоит сравнить соотношения (29) и (30) в нашей теореме 1 с пунктом (iii) а) в теореме 1 из [21]). Однако часть утверждений теоремы 1 из [21] не охватывается нашей теоремой 1, поскольку они вовлекают асимптотические оценки для моментов численностей частиц в терминах локальных времен уровня $w$ марковской цепи $\eta$. При этом авторы статьи [21] не обсуждают асимптотическое поведение таких локальных времен.

П р и м е р 3. Сосредоточимся на ветвящемся случайном блуждании по $\mathbf{Z}^{d}, d \in \mathbf{N}$, с несколькими источниками ветвления, исследованном в [34]. Эта модель является частным случаем КВП таким, что $\eta$ представляет собой симметричное и пространственно-однородное случайное блуждание по $\mathbf{Z}^{d}$ с конечной дисперсией скачков, причем симметрия случайного блуждания может нарушаться только в конечном множестве точек из $\mathbf{Z}^{d}$. Как установлено в [34], скорость экспоненциального роста численностей частиц является наибольшим положительньм числом $\lambda$, удовлетворяющим уравнению $\operatorname{det} \widehat{D}(\lambda)=0$ (что согласуется с нашей леммой 5). Однако необходимые и достаточные условия существования этого положительного решения $\lambda$ не были найдены, как сказано в заключении статьи [34]. Стоит подчеркнуть, что такое необходимое и достаточное условие приводится в настоящей статье и в силу леммы 5 и теоремы 1 состоит в том, что $\rho(D)>1$, т.е. в наших терминах заключается в надкритичности режима КВП.

Завершая статью, хотелось бы отметить, что наш подход сочетания техники времен достижения с запретом и аппарата вспомогательных многотипных процессов Беллмана-Харриса позволяет получить и обосновать классификацию каталитических ветвящихся процессов с произвольным конечным множеством катализаторов. Результаты данной статьи справедливы при минимальных ограничениях, налагаемых на характер перемещения и размножения частиц. Поэтому эти результаты обобщают ряд предшествующих работ на близкие темы. Развитый подход может быть использован в последующем изучении других характеристик каталитических ветвящихся процессов. 
Автор признателен профессорам В.А. Ватутину и М.А. Лифшицу за полезные обсуждения. Автор также благодарен рецензенту за ценные замечания, направленные на улучшение изложения статьи.

\section{СПИСОК ЛИТЕРАТУРЫ}

1. Булинская E. Вл. Времена достижения с запретом для случайного блуждания. Матем. тр., 2012, т. 15, № 1, с. 3-26.

2. Булинская E. Вл. Докритическое каталитическое ветвящееся случайное блуждание с конечной или бесконечной дисперсией числа потомков. - Тр. МИАН, 2013, т. 282 , c. $69-79$.

3. Ватутин $B . A$. Ветвящиеся процессы с финальными типами частиц и случайные деревья. - Теория вероятн. и ее примен., 1994, т. 39, в. 4, с. 699-715.

4. Ватутин B. A., Топчий B. A. Каталитические ветвящиеся случайные блуждания на $\mathbf{Z}^{d}$ с ветвлением в нуле. - Матем. тр., 2011, т. 14, № 2, с. 28-72.

5. Ватутин B.A., Топчий B.A. Критические ветвящиеся процессы БеллманаХарриса с долго живущими частицами. - Тр. МИАН, 2013, т. 282, с. 257-287.

6. Гантмахер Ф.Р. Теория матриц. М.: ФИЗМАТЛИТ, 2010, 560 с.

7. Лифшии M. А. Циклическое поведение максимума в иерархической схеме суммирования. - Зап. научн. сем. ПОМИ, 2012, т. 408, с. 268-284.

8. Севастьянов Б. А. Ветвящиеся процессы. М.: Наука, 1971, 436 с.

9. Чжун К. Л. Однородные цепи Маркова. М.: Мир, 1964, 425 с.

10. Яровая Е.Б. Критерии экспоненциального роста числа частиц в моделях ветвящихся случайных блужданий. - Теория вероятн. и ее примен., 2010, т. 55, в. 4, c. $705-731$.

11. Albeverio S., Bogachev L. V. Branching random walk in a catalytic medium. I. Basic equations. - Positivity, 2000, v. 4, № 1, p. 41-100.

12. Albeverio S., Bogachev L.V., Yarovaya E. B. Asymptotics of branching symmetric random walk on the lattice with a single source. - C. R. Math. Acad. Sci. Paris, 1998, v. 326, № 8, p. 975-980.

13. Barbour A.D., Luczak M.J. Central limit approximations for Markov population processes with countably many types. - Electron. J. Probab., 2012, v. 17, №90, p. 1-16.

14. Bertacchi D., Zucca $F$. Characterization of critical values of branching random walks on weighted graphs through infinite-type branching processes. - J. Statist. Phys., 2009, v. 134, № 1, p. 53-65.

15. Biggins J.D. Spreading speeds in reducible multitype branching random walk. Ann. Appl. Probab., 2012, v. 22, № 5, p. 1778-1821.

16. Bulinskaya E. Vl. Local particles numbers in critical branching random walk. - J. Theoret. Probab., 2014, v. 27, № 3, p. 878-898.

17. Bulinskaya E. Vl. Finiteness of hitting times under taboo. - Statist. Probab. Lett., 2014, v. 85 , p. $15-19$.

18. Carmona Ph., Hu Y. The spread of a catalytic branching random walk. - Ann. Inst. Henri Poincaré Probab. Statist., 2014, v. 50, № 2, p. 327-351.

19. Crump K.S. On systems of renewal equations: The reducible case. - J. Math. Anal. Appl., 1970, v. 31, № 3, p. 517-528.

20. Dawson D., Fleischmann K. A super-Brownian motion with a single point catalyst. Stochastic Process. Appl., 1994, v. 49, № 1, p. 3-40.

21. Döring L., Roberts $M$. Catalytic branching processes via spine techniques and renewal theory. - Lecture Notes in Math., 2013, v. 2078, p. 305-322.

22. Fleischmann K., Le Gall J.-F. A new approach to the single point catalytic superBrownian motion. - Probab. Theory Related Fields, 1995, v. 102, № 1, p. 63-82.

23. Hautphenne S., Latouche G., Nguyen G.T. Extinction probabilities of branching processes with countably infinitely many types. - Adv. Appl. Probab., 2013, v. 45, № 4, p. 1068-1082. 
24. $H u$ Y., Shi Z. Minimal position and critical martingale convergence in branching random walks, and directed polymers on disordered trees. - Ann. Probab., 2009, v. 37 , № 2 , p. $742-789$.

25. Jones $G$. Calculations for multi-type age-dependent binary branching processes. J. Math. Biol., 2011, v. 63, № 1, p. 33-56.

26. Good I. J. The multivariate saddlepoint method and chi-squared for the multinomial distribution. - Ann. Math. Statist., 1961, v. 32, № 2, p. 535-548.

27. Mode Ch.J. A multidimensional age-dependent branching process with applications to natural selection. I. - Math. Biosci., 1968, v. 3, p. 1-18.

28. Mode Ch. J. A multidimensional age-dependent branching process with applications to natural selection. II. - Math. Biosci., 1968, v. 3, p. 231-247.

29. Moy S.-T. C. Extensions of a limit theorem of Everett, Ulam and Harris on multitype branching processes to a branching process with countably many types. - Ann. Math. Statist., 1967, v. 38, № 4, p. 992-999.

30. Sagitov $S$. Linear-fractional branching processes with countably many types. Stochastic Process. Appl., 2013, v. 123, № 8, p. 2940-2956.

31. Seneta E. Non-negative Matrices and Markov Chains. New York: Springer, 2006, $287 \mathrm{p}$

32. Vatutin V.A., Topchii V.A., Yarovaya E. B. Catalytic branching random walk and queueing systems with random number of independent servers. - Theory Probab. Math. Statist., 2004, № 69, p. 1-15.

33. Yakovlev A. Y., Yanev N. M. Relative frequencies in multitype branching processes. Ann. Appl. Probab., 2009, v. 19, № 1, p. 1-14.

34. Yarovaya E. B. Branching random walks with several sources. - Math. Popul. Stud., 2013, v. 20, № 1, p. 14-26. 